\title{
Characterization of the human Activin-A receptor type II-like kinase 1 (ACVRL1) promoter and its regulation by $\mathrm{Sp} 1$
}

Eva M Garrido-Martin ${ }^{1}$, Francisco J Blanco ${ }^{1}$, Africa Fernandez-L ${ }^{1,2}$, Carmen Langa ${ }^{1}$, Calvin P Vary ${ }^{3}$, Ursula E Lee ${ }^{4}$, Scott L Friedman ${ }^{4}$, Luisa M Botella ${ }^{1}$, Carmelo Bernabeu ${ }^{1 *}$

\begin{abstract}
Background: Activin receptor-like kinase 1 (ALK1) is a Transforming Growth Factor- $\beta$ (TGF- $\beta$ ) receptor type I, mainly expressed in endothelial cells that plays a pivotal role in vascular remodelling and angiogenesis. Mutations in the ALK1 gene (ACVRL1) give rise to Hereditary Haemorrhagic Telangiectasia, a dominant autosomal vascular dysplasia caused by a haploinsufficiency mechanism. In spite of its patho-physiological relevance, little is known about the transcriptional regulation of ACVRL1. Here, we have studied the different origins of ACVRL1 transcription, we have analyzed in silico its 5'-proximal promoter sequence and we have characterized the role of Sp1 in the transcriptional regulation of ACVRL1.
\end{abstract}

Results: We have performed a 5'Rapid Amplification of cDNA Ends (5'RACE) of ACVRL1 transcripts, finding two new transcriptional origins, upstream of the one previously described, that give rise to a new exon undiscovered to date. The 5'-proximal promoter region of ACVRL1 $(-1,035 /+210)$ was analyzed in silico, finding that it lacks TATA/ CAAT boxes, but contains a remarkably high number of GC-rich Sp1 consensus sites. In cells lacking Sp1, ACVRL1 promoter reporters did not present any significant transcriptional activity, whereas increasing concentrations of Sp1 triggered a dose-dependent stimulation of its transcription. Moreover, silencing Sp1 in HEK293T cells resulted in a marked decrease of ACVRL1 transcriptional activity. Chromatin immunoprecipitation assays demonstrated multiple Sp1 binding sites along the proximal promoter region of ACVRL1 in endothelial cells. Furthermore, demethylation of $C p G$ islands, led to an increase in ACVRL1 transcription, whereas in vitro hypermethylation resulted in the abolishment of Sp1-dependent transcriptional activation of ACVRL1.

Conclusions: Our results describe two new transcriptional start sites in ACVRL1 gene, and indicate that Sp1 is a key regulator of $A C V R L 1$ transcription, providing new insights into the molecular mechanisms that contribute to the expression of ACVRL1 gene. Moreover, our data show that the methylation status of CpG islands markedly modulates the Sp1 regulation of ACVRL1 gene transcriptional activity.

\section{Background}

ALK1 (Activin receptor-Like Kinase 1) is a transmembrane type I receptor of the Transforming Growth Factor $-\beta$ (TGF- $\beta$ ) superfamily of ligands, mainly found in endothelial cells. Its expression has been reported not only in highly vascularized tissues including lung, placenta, and heart $[1,2]$, but also at specific sites of

\footnotetext{
* Correspondence: bernabeu.c@cib.csic.es

'Centro de Investigaciones Biológicas (CIB), Consejo Superior de Investigaciones Cientificas (CSIC) and Centro de Investigación Biomédica en Red de Enfermedades Raras (CIBERER), Ramiro de Maeztu 9, 28040 Madrid, Spain
}

(c) 2010 Garrido-Martin et al; licensee BioMed Central Ltd. This is an Open Access article distributed under the terms of the Creative Commons Attribution License (http://creativecommons.org/licenses/by/2.0), which permits unrestricted use, distribution, and reproduction in any medium, provided the original work is properly cited. epithelial-mesenchymal interactions [3], and in other cell types such as monocytes [4], microglia [5], skin fibroblasts [6], stellate hepatic cells [7], chondrocytes [8], neural crest stem cells [9] and more recently myoblasts [10]. Nonetheless, most studies to date suggest that its major roles are related to the endothelial specific expression pattern. ALK1 is involved in angiogenesis $[11,12]$, and there is growing evidence indicating that it plays a key function in the arterial/venous differentiation during embryonic vascular development $[13,14]$. It has been reported that ALK1 interacts with three ligands: with TGF- $\beta 1$ and TGF- $\beta 3$, in complex with the receptor 
type II (T $\beta$ R-II) [15]; and with Bone Morphogenetic Protein 9 (BMP9), in complex with the Activin Receptor type IIA (ActRIIA) or the BMP receptor type II (BMPRII) [16]. In the endothelium, circulating TGF- $\beta$ signals from the lumen of the vascular vessel to the cytoplasm of the endothelial cell by interacting with its specific receptor complex. This complex consists of three different dimeric proteins: receptor type II (T $\beta R$ II), receptor type I (T $\beta \mathrm{R}-\mathrm{I})$ and an ancillary co-receptor (T $\beta$ R-III: Betaglycan or Endoglin) [17]. ALK5 is the predominant T $\beta R-I$ in the majority of the cell types, but in endothelial cells ALK1 shares the T $\beta$ R-I function with ALK5 in vitro. The significance of this apparent redundancy is explained because ALK1 and ALK5 signal in opposite directions, balancing the TGF- $\beta$ signalling pathway in this cell type [11]. ALK5 is able to arrest the cell growth, leading to a differentiated state in the maturation phase of angiogenesis, with formation of new extracellular matrix around the new vessel formed. ALK1 appears to play opposite physiological functions, since it is responsible for the events occurring during the activation phase of angiogenesis, including metalloprotease activation, proliferation of endothelial cells, and inhibition of differentiation [18]. Thus, these complementary effects are mediated through different target genes of the two signalling pathways. ALK5 signals through Smad2/3 to regulate PAI-1 (Plasminogen Activator Inhibitor-1), Collagen I, or NOS-3/eNOS (Endothelial Nitric Oxide Synthase), whereas ALK1 signals through Smad1/5/8 to induce genes involved in proliferation such as $I d 1$ (Inhibitor of differentiation 1), Id2 (Inhibitor of differentiation 2), Smad6, Smad7, ENG (Endoglin) or BMPRII [19]. In addition, it has been shown that BMP9 is a quiescence factor for the microvasculature [20].

The gene encoding ALK1 (ACVRL1, Activin-A receptor type II-like kinase 1) spans $15,943 \mathrm{bp}$ within the large arm of chromosome 12 (12q11-q14), coded on the positive strand (GeneID: 94). ACVRL1 cDNA was described first in 1993 simultaneously by two different groups showing a characteristic transcriptional start site (TSS), where the first exon is transcribed but not translated. To date, two different variants of mRNA transcripts have been described: variant 1 [GenBank: NM_000020.2] [21] and variant 2 [GenBank: NM_001077401.1] [22], both encoding the same ALK1 protein of 503 amino acids.

Mutations in ACVRL1 give rise to an autosomal vascular dysplasia called Hereditary Haemorrhagic Telangiectasia type 2 (HHT2) [1], while HHT type 1 is caused by mutations in ENG, the gene coding for the TGF- $\beta$ co-receptor Endoglin [23]. HHT1 and HHT2 are genetically dominant and mutant homozygosis is lethal, as confirmed in knock out mice for ENG and ACVRL1
[24-26]. Current estimates suggest that one in 5,0008,000 people are affected by HHT [27]. Patients are clinically diagnosed according to the Curaçao criteria [28], including nose bleeds, mucocutaneous telangiectases, internal arteriovenous malformations and familial inheritance. Around $90 \%$ of HHT patients have been genetically diagnosed as HHT1 or HHT2, and multiple mutations along exonic regions of $A C V R L 1$ and ENG have been described [29]. Because approximately $10 \%$ of HHT patients clinically diagnosed have unidentified mutations, the study of intronic sequences, splice sites and promoter regions of both genes is of critical importance. Moreover, haploinsufficiency is currently accepted as the basis for the pathogenicity of HHT [29] and therefore the understanding of the transcriptional mechanisms and the assessment of ways to increase the transcription rate may be crucial to identify strategies to counteract haploinsufficiency. While several reports have already described the organization and control of ENG promoter [30-33], very little has been done in relation to the $A C V R L 1$ promoter. In this work, we have analyzed the 5 '-proximal $A C V R L 1$ promoter region, characterized new transcriptional initiation points, and analyzed the transcriptional mechanisms that regulate its expression.

\section{Results}

Identification of novel TSSs for human ACVRL1 gene revealed by 5'RACE

In order to characterize the origins of $A C V R L 1$ transcription in endothelial cells, a $5^{\prime} \mathrm{RACE}$ of total RNA obtained from HUVECs was performed. After nested PCR, the amplified bands were cloned and sequenced (Figure 1A and 1B). The three major products amplified correspond to three different 5 'flanking regions. More than $50 \%$ of the clones corresponded to the mRNA1 of ACVRL1 previously described [21], whereas two new TSSs (mRNA3 and mRNA4) were also identified in several clones from independent experiments (Figure 1B). Interestingly, both alternative mRNAs begin the transcription upstream the TSS $(+1)$, in the positions -510 and -470 , respectively. Accordingly, the exons have been renumbered in this work in such a way that the new exon 1 is upstream of the previously described TSS $(+1)$. The numbering of the TSS $(+1)$ has been maintained because it appears to be the predominant TSS. Thus, the previously considered exon 1 turns into exon 2, being named 2A, in the case of NM_000020.2 and 2B in the case of NM_001077401. Likewise, the newly discovered upstream exon now becomes exon 1, named $1 \mathrm{~A}$ in the mRNA3, or $1 \mathrm{~B}$, in the variant mRNA4.

Figure $1 C$ shows the scheme of all the ACVRL1 transcript variants found in this work (mRNA1, 3 and 4) and in previous reports (mRNA1 and 2). The variant 
A
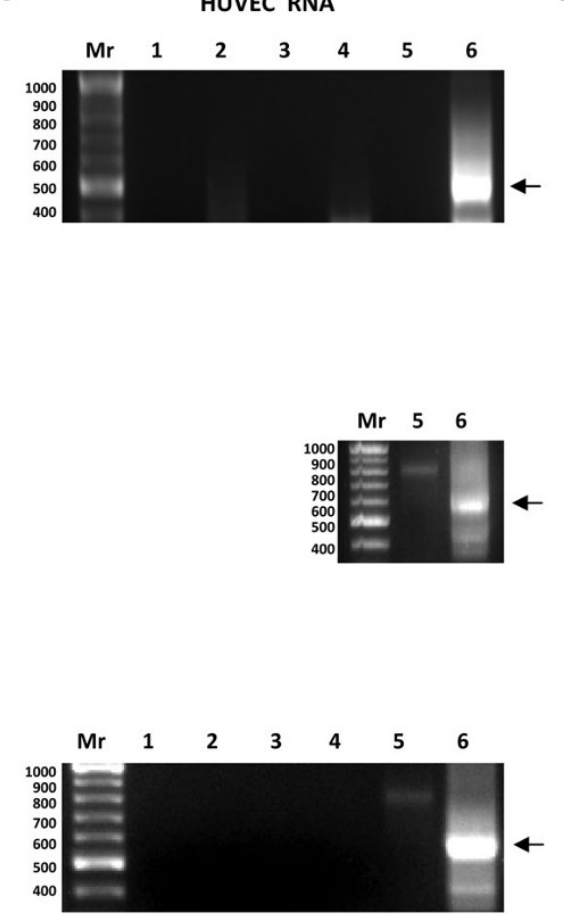

C
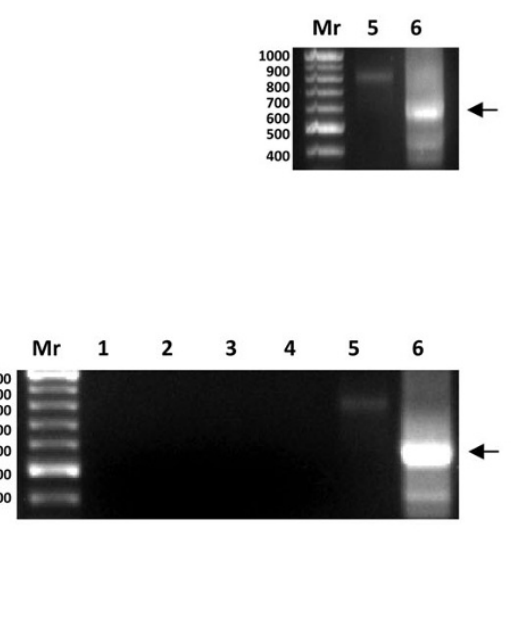

B

mRNA1 $(585$ bp)

CGCGGATCCGAACACTGGGTTTGCTGGCTTTGATGAGG

Adapter (inner primer)

CTGCGGCCGCGCGGTGGAGGGGAGGTGGCCCCGGTCCGCCGAAGGCTAGCGCCCCGCCACCCGCAGAGCGGGCCCAGÄ
$+\mathbf{2 7 8}+5053$

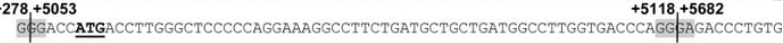

AAGCCGTCTCGGGGCCCGCTGGTGACCTGCACGTGTGAGAGCCCACATTGCAAGGGGCCTACCTGCCGGGGGGCCTGG

TGCACAGTAGTGCTGGTGCGGGAGGAGGGGAGGCACCCCCAGGAACATCGGGGCTGCGGGAACTTGCACAGGGAGCTC TGCAGGGGGCGCCCCACCGAGTTCGTCAACCACTACTGC Inner specific pALK1 primer $(+5869 /+5887)$

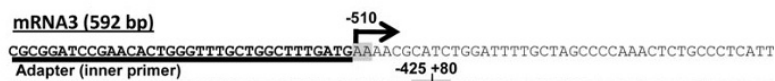

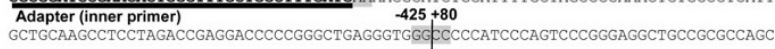
TGCGCCGAGCGAGCCCCTCCCCGGCTCCAGCCCGGTCCGGGGCCGCGCCCGGACCCCAGCCCGCCGTCCAGCGCTGGC GGTGCAACTGCGGCCGCGCGGTGGAGGGGAGT GGCCCCGGTCCGCCGAAGGCTAGCGCCCCGCCACCCGCAGAGCGG
$+\mathbf{2 7 8}+5053$
$\mathbf{+ 5 1 1 8 + 5 6 8 2}$

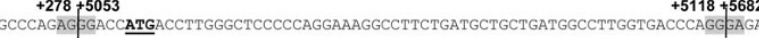
CCCTGTGAAGCCGTCTCGGGGCCCGCTGGTGACCTGCACGTGTGAGAGCCCACATTGCAAGGGGCCTACCTGCCGGG GGCCTGGTGCACAGTAGTGCTGGTGCGGGAGGAGGGGAGGCACCCCCAGGAACATCGGGGCTGCGGGAACTTGCACAG GGAGCTCTGCAGGGGGCGCCCCACCGAGTTCGTCAACCACTACTGC
Inner specific pALK1primer (+5869/+5887)

mRNA4 (552 bp) CGCGGATCCGAACACTGGGTTTGCTGGCTTTGATGATTGCTGCAAGCCTCCTAGACCGAGGACCCCCGGGCTGAGGGT $\frac{-425+80}{G G G C C C C A T C C C A G T C C C G G G A G G C T G C C G C G C C A G C T G C G C C G A G C G A G C C C C T C C C C G G C T C C A G C C C G G T C C G C ~}$ GGCCGCGCCCGGACCCCAGCCCGCCGTCCAGCGCTGGCGGTGCAACTGCGGCCGCGCGGTGGAGGGGAGGTGGCCCCG GTCCGCCGAAGGCTAGCGCCCCGCCACCCGCAGAGCGGGCCCAGÄ $\$ 5$ GACCATGACCTTGGGCTCCCCCAGGAAAGGC CTTCTGATGCTGCTGATGGCCTTGGTGACCCAGGFAGACCCTGTGAAGCCGTCTCGGGGCCCGCTGGTGACCTGCACE TGTGAGAGCCCACATTGCAAGGGGCCTACCTGCCGGGGGGCCTGGTGCACAGTAGTGCTGGTGCGGGAGGAGGGGAGG CACCCCCAGGAACATCGGGGCTGCGGGAACTTGCACAGGGAGCTCTGCAGGGGGCGCCCCACCGAGTTCGTCAACCAC $\frac{\text { TACTGC }}{+5887)}$

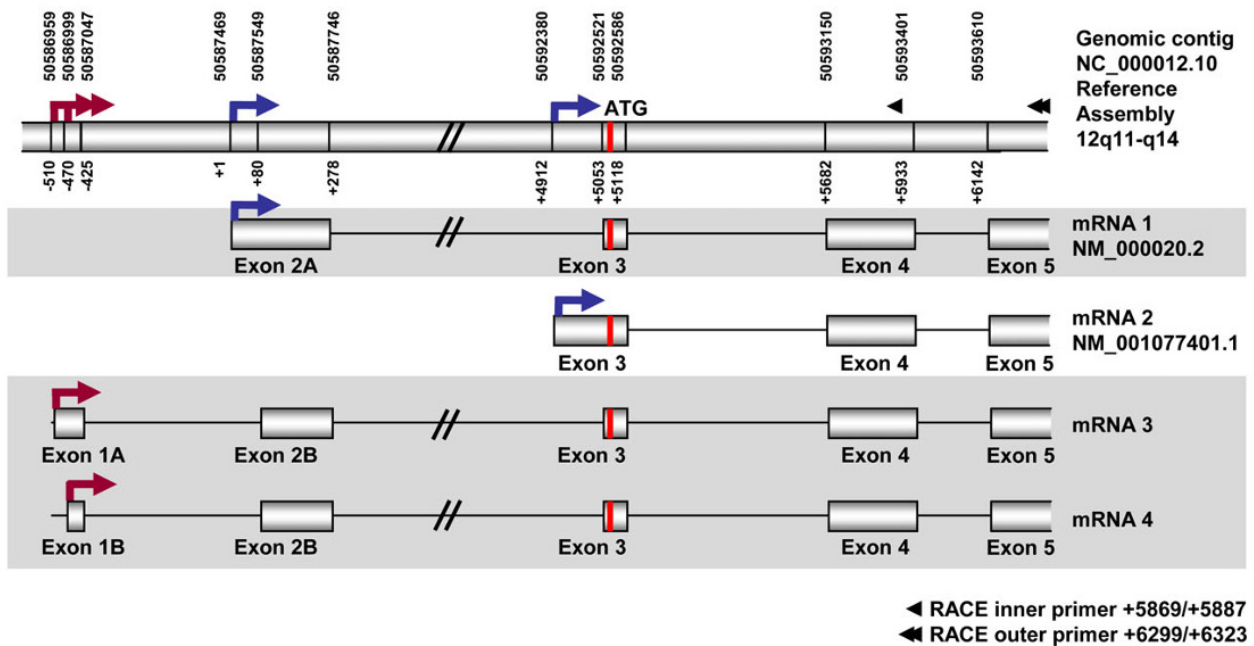

Figure $15^{\prime}$ Rapid Amplification of CDNA Ends (5'RACE) of ACVRL1 transcripts from HUVECs. Electrophoretic analysis of nested PCR amplification products. Lanes: 1 and 2 (without template; outer and inner nested PCRs), 3 and 4 (RNA without CIP/TAP treatment; outer and inner), 5 and 6 (CIP/TAP treated RNA; outer and inner). More than 10 different experiments were performed and three representative gels are shown. (B) Nucleotide sequence of the products. Primers used are underlined. Grey boxes indicate the junctions between different exons. The predominant transcript found in HUVECs corresponds to the mRNA1 previously described in placenta. Two novel variants described in this work have been named mRNA3 [GenBank:HM161905] and mRNA4 [GenBank:HM161906]. Numbers are given according to the genomic sequence from +1 TSS. Fragments starting from -510 of mRNA3 and from -470 of mRNA4 are the newly observed transcribed regions. The sequences of these three isoforms are identical downstream from +79 . (C) Schematic representation of the $5^{\prime}$ flanking region of ACVRL 1 transcripts. The previously described mRNA1 [GenBank:NM 000020.2] and mRNA2 [GenBank:NM 001077401.1], and mRNA3 and mRNA4 found in this work are shown. All ACVRL1 encoded proteins found to date have the same 503 amino acid sequence. mRNA1 has been found with the same characteristics as previously described. Taking into account the new sequence, ACVRL1 rearranges from 10 to 11 exons; starting the new exon 1 at -510 (variant 1A) or -470 (variant 1B). Also, previously described exon 1 is renamed as exon 2 (variants 2A and 2B). Red arrows represent the two newly described TSSs. Blue arrows represent the two TSSs previously known. 
mRNA1 begins in the position +1 , followed by an exon of $278 \mathrm{bp}$ (exon 2A) and then upon splicing of the longest intron $(\sim 5 \mathrm{~kb})$ of $A C V R L 1$, it continues with an exonic sequence containing the ATG start codon (exon $3)$. The mRNA2 has no exon $2 \mathrm{~A}$, and its exon 3 is longer in its 5 'region, containing an additional $141 \mathrm{bp}$ fragment. The starting ATG is located at the beginning of exon $3(+5,058 /+5,060)$, coinciding with mRNA1, therefore, both transcripts give rise to the same 503 amino acid protein.

The fragments obtained in the present work correspond to isoforms mRNA3 and mRNA4. The corresponding cDNA sequences have been deposited in GenBank database with accession numbers HM161905 and HM161906, respectively. Both of them reveal a cryptic exon placed upstream the +1 , resulting in two, instead of one, transcribed but not translated exons. This event gives rise to a rearrangement from 10 to 11 exons in the composition of the coding region of ACVRL1.

\section{The human ACVRL1 promoter lacks TATA/CAAT boxes and has multiple $\mathrm{Sp} 1$ motifs}

Human ACVRL1 gene resides on chromosome 12q11q14 [34]. Thus, a 1,244 bp fragment comprising from position $-50,586,434$ to position $-50,587,679$ of the contig [GenBank:NC_000012.10, Reference assembly] was amplified by PCR from human genomic DNA (Figure 2). This fragment corresponds to positions from $-1,035$ bp to +210 bp relative to the $(+1)$ TSS. Interestingly, this $5^{\prime}$-proximal regulatory region of ACVRL1 lacks TATA and CAAT boxes. A predictive in silico analysis of $A C V R L 1$ promoter using the Genomatix MatInspector software tool revealed a very high number of GC-rich regions and multiple putative Sp1 binding consensus sites (Table 1). Fourteen different possible sites for Sp1 interaction were found, located in both strands: Forward strand (Sites $-810 /-796$, $-734 /-720,-728 /-714,-524 /-510,-435 /-421,-88 /-74$, $-70 /-56$ and $+15 /+30$ ); and reverse strand (Sites $-919 /-$ $905,-411 /-397,-401 /-387,-309 /-295,-184 /-170$ and + $124 /+139)$. All of these sites have a very high similarity with the theoretical matrix (score $>0.85$ ). Three AP1 and four AP4 sites were found dispersed along the sequence. In addition, the $5^{\prime}$-flanking sequence of $A C V R L 1$ gene contains several putative regulatory elements also present in other endothelial genes (see Figure 2 and Table 1). These consensus elements include eight motifs for the Ets (E26-Transformation-Specific) family of transcription factors, 23 sequences sensitive for KLF (Krüppel-Like Factor) recognition, seven NF $\kappa \mathrm{B}$ (Nuclear Factor kappa-light-chain-enhancer of activated $\mathrm{B}$ cells) motifs, five E2F (Elongation Factor 2) consensus sequences, one Smad binding element (SBE) and seven sites for RXR (Retinoid X Receptor) dimers. Moreover, three HIF (Hypoxia Inducible Factor) consensus sequences were found distributed along the $A C V R L 1$ promoter sequence.

\section{The ACVRL1 promoter region is highly conserved among different species}

The ACVRL1 proximal promoter sequence has several features that suggest a high level of regulation. One of these features is the possibility to begin the transcription in different points. To evaluate the importance of each putative regulatory site, the ACVRL1 promoter of several mammalian species were compared using the ClustalW2 algorithm. The species analyzed included mouse, rat, cow, orangutan, rhesus monkey, horse, chimpanzee and dog. For the alignments, we selected the same region than the one we were studying in humans (from $-1,035 \mathrm{bp}$ to $+210 \mathrm{bp}$ of the TSS). Figure 3 shows the comparative alignment of the ACVRL1 human sequence surrounding the different TSSs with the same regions in other animal species. Panel A shows a scheme of the regions aligned in panel $\mathrm{B}$. The previously described TSS (identified by the +1 ) has a highly conserved sequence among primates (AGGAAACGG), suggesting an important functional role. Moreover, the two newly described TSSs are conserved only in this group of species $(-510$ bp AAAACGC in exon $1 \mathrm{~A}$ and $-470 \mathrm{bp}$ ATTGCT in exon 1B). Of note, both sequences at the beginning of exons $1 \mathrm{~A}$ and $1 \mathrm{~B}$ are less conserved than the region surrounding TSS $(+1)$.

Another interesting feature about the putative $A C V R L 1$ promoter is the high number of $\mathrm{Sp} 1$ consensus sites along the sequence, and near to the TSSs. These sites are important as possible key regulators for ACVRL1 transcription, so we compared these motifs among different species. The scheme of $\mathrm{Sp} 1$ sites present in the sequence is shown in Figure 4A. The alignments of these fragments and the same regions from other animal species are included in Figure 4B. Of note, these sites appear highly conserved, especially between humans and other primates, which is a characteristic of promoters under Sp1 regulation and lacking TATA/ CAAT boxes.

\section{Basal activity of $A C V R L 1$ promoter suggests the presence} of possible positive and negative regulatory regions

To assess the transcriptional activity of the ACVRL1 promoter fragment, it was cloned into a luciferase reporter vector. A schematic representation of the ACVRL1 promoter fragment cloned into pGL2-luc, with the locations of the different TSS and the ATG translation start codon, is shown in Figure 5A. Serial 5'-deleted constructs from $-1,035$ bp to -284 bp of the ACVRL1 proximal promoter were generated (Figure $5 \mathrm{~B}$, left) and their basal 
transcription activity assessed in HMEC-1. In this endothelial cell line, the shortest construct maintains the majority of the transcriptional activity, whereas the $-422 /$ +59 construct displays one third of the activity compared with the whole sequence (Figure 5B, right). In order to unravel if $A C V R L 1$ could be transcriptionally regulated by its own ligands, the effect of TGF- $\beta 1$ or BMP9 was assessed. HMEC-1 cells were transfected with different pALK1 reporter constructs and treated with either TGF$\beta 1$ or BMP9 using the time and dose previously established in endothelial cells [35]. None of these treatments had a significant effect on the transcriptional activity of the reporter constructs, except for the construct $-422 /+59$, whose basal activity seems to be slightly upregulated in the presence of BMP9 (Figure 5C).

\section{Sp1 is critical for the ACVRL1 basal transcription}

Since 14 putative target sequences for Sp1 were predicted in the ACVRL1 promoter, their contribution to the basal activity was assessed in Schneider Drosophila 
Table 1 Main putative transcription factor binding sites found in the ACVRL1 promoter region

\begin{tabular}{|c|c|c|c|c|c|c|c|c|c|c|c|}
\hline Family & Start pos. & End pos. & Str. & Matrix sim. & Core sim. & Family & Start pos. & End pos. & Str. & Matrix sim. & Core sim. \\
\hline \multirow[t]{3}{*}{ AP1 } & -875 & -855 & - & 0.823 & 1.0 & HIF & -808 & $\begin{array}{l}-792 \\
\end{array}$ & + & 0.85 & 0.9 \\
\hline & -873 & -853 & + & 0.899 & 0.813 & & -286 & -274 & + & 0.885 & 1.0 \\
\hline & -871 & -851 & - & 0.994 & 1.0 & & -236 & -219 & - & 0.843 & 0.75 \\
\hline \multirow[t]{4}{*}{ AP4 } & -881 & -865 & - & 0.923 & 1.0 & NFKB & -851 & -839 & + & 0.901 & 1.0 \\
\hline & -820 & -804 & - & 0.87 & 0.882 & & -786 & -774 & + & 0.852 & 1.0 \\
\hline & -506 & -490 & - & 0.961 & 1.0 & & -784 & -772 & - & 0.919 & 1.0 \\
\hline & +179 & +195 & - & 0.855 & 1.0 & & -534 & -522 & + & 0.985 & 1.0 \\
\hline CEBP & -432 & -418 & + & 0.945 & 0.971 & & -533 & -521 & - & 0.947 & 1.0 \\
\hline \multirow[t]{14}{*}{ EKLF/KLF1 } & -918 & -902 & - & 0.924 & 1.0 & & -220 & -208 & + & 0.84 & 1.0 \\
\hline & -886 & -870 & - & 0.911 & 1.0 & & -158 & -146 & + & 0.838 & 1.0 \\
\hline & -808 & -792 & + & 0.975 & 1.0 & RXR & -979 & -955 & + & 0.954 & 1.0 \\
\hline & -737 & -721 & + & 0.932 & 1.0 & & -871 & -847 & + & 0.758 & 0.771 \\
\hline & -731 & -715 & + & 0.917 & 1.0 & & -864 & -840 & + & 0.818 & 0.824 \\
\hline & -433 & -417 & + & 0.977 & 1.0 & & -535 & -511 & + & 0.847 & 0.952 \\
\hline & -410 & -394 & - & 0.933 & 1.0 & & -441 & -417 & + & 0.763 & 0.812 \\
\hline & -405 & -389 & - & 0.95 & 1.0 & & -314 & -290 & - & 0.819 & 0.81 \\
\hline & -313 & -297 & - & 0.974 & 1.0 & & -230 & -206 & - & 0.791 & 0.905 \\
\hline & -241 & -225 & - & 0.916 & 1.0 & SMAD & -270 & -262 & + & 0.969 & 1.0 \\
\hline & -107 & -91 & + & 0.923 & 1.0 & SP1 & -919 & -905 & - & 0.901 & 0.807 \\
\hline & -73 & -57 & + & 0.916 & 1.0 & & -810 & -796 & + & 0.885 & 0.872 \\
\hline & -68 & -52 & + & 0.971 & 1.0 & & -734 & -720 & + & 0.997 & 1.0 \\
\hline & +125 & +141 & - & 0.947 & 1.0 & & -728 & -714 & + & 0.879 & 1.0 \\
\hline \multirow[t]{8}{*}{ ETS } & -939 & -919 & + & 0.961 & 1.0 & & -524 & -510 & + & 0.913 & 1.0 \\
\hline & -797 & -777 & + & 0.922 & 1.0 & & -435 & -421 & + & 0.881 & 0.872 \\
\hline & -791 & -771 & + & 0.965 & 1.0 & & -411 & -397 & - & 0.89 & 0.807 \\
\hline & -758 & -738 & - & 0.943 & 1.0 & & -401 & -387 & - & 0.895 & 0.872 \\
\hline & -699 & -679 & + & 0.972 & 1.0 & & -309 & -295 & - & 0.891 & 0.872 \\
\hline & -388 & -368 & - & 0.945 & 1.0 & & -184 & -170 & - & 0.98 & 1.0 \\
\hline & -373 & -353 & - & 0.914 & 1.0 & & -88 & -74 & + & 0.885 & 1.0 \\
\hline & +36 & +56 & + & 0.927 & 1.0 & & -70 & -56 & + & 0.892 & 0.872 \\
\hline \multirow[t]{5}{*}{$\mathrm{E} 2 \mathrm{~F}$} & -493 & -477 & + & 0.849 & 1.0 & & +15 & +30 & + & 0.987 & 1.0 \\
\hline & -143 & -127 & - & 0.861 & 0.81 & & +124 & +139 & - & 0.93 & 0.877 \\
\hline & +98 & +114 & - & 0.971 & 1.0 & ZF9/KLF6 & -815 & -793 & - & 0.891 & 0.923 \\
\hline & +99 & +115 & + & 0.964 & 1.0 & & -739 & -717 & - & 0.899 & 1.0 \\
\hline & +150 & +166 & - & 0.875 & 1.0 & & -148 & -126 & - & 0.881 & 1.0 \\
\hline \multirow[t]{3}{*}{ GKLF/KLF4 } & -981 & -969 & + & 1.000 & 0.862 & & -109 & -87 & - & 0.897 & 0.821 \\
\hline & -600 & -588 & + & 1.000 & 0.883 & & -86 & -64 & - & 0.879 & 1.0 \\
\hline & -3 & +9 & + & 0.770 & 0.868 & & +150 & +172 & + & 0.936 & 1.0 \\
\hline
\end{tabular}

Note: Start/End pos, starting/ending position of the consensus site in the sequence; Str, Strand-sense; Matrix sim, Matrix (groups of functionally similar transcription factors) similarity factor (0-1); Core sim, Core consensus sequence (4 highest conserved positions) similarity factor (0-1); AP1, Activator Protein 1; AP4, Activator Protein 4; CEBP, CCAAT/Enhancer Binding Protein; EKLF, Erythroid Krüppel-Like Factor; ETS, E26-Transformation-Specific transcription factor; E2F, Elongation Factor 2; GKLF, Gut-related Krüppel-Like Factor; HIF, Hypoxia Inducible Factor; NF?B, Nuclear Factor of kappa light polypeptide gene enhancer in Bcells; RXR, Retinoid X Receptor; SMAD, Sma and Mad-related; SP1, Specificity Protein 1; ZF9, Krüppel-like Zinc Finger protein 9.

embryonic S2 cells. These cells are undifferentiated and do not express endogenous Sp1 or any other related member of its family. As shown in Figure 6A, left, the proximal promoter of $A C V R L 1$ shows background levels of basal activity in the absence of Sp1. By contrast, ectopic expression of Sp1 shows a dramatic effect on pALK1 activity. Even with very low amounts of the transfected
Sp1 expression vector, the basal transcription of pALK1 is increased $\sim 100$ fold following a dose-response curve. There is a saturating effect around $25 \mathrm{ng} \mathrm{Sp} 1$ where the promoter is stimulated $\sim 500$ fold. The same experiment performed in HEK293T cells resulted in the stimulation of the basal activity up to 3 -fold (Figure 6A, right). This moderate increase, as compared to S2 cells ( $\sim 500$ fold), 


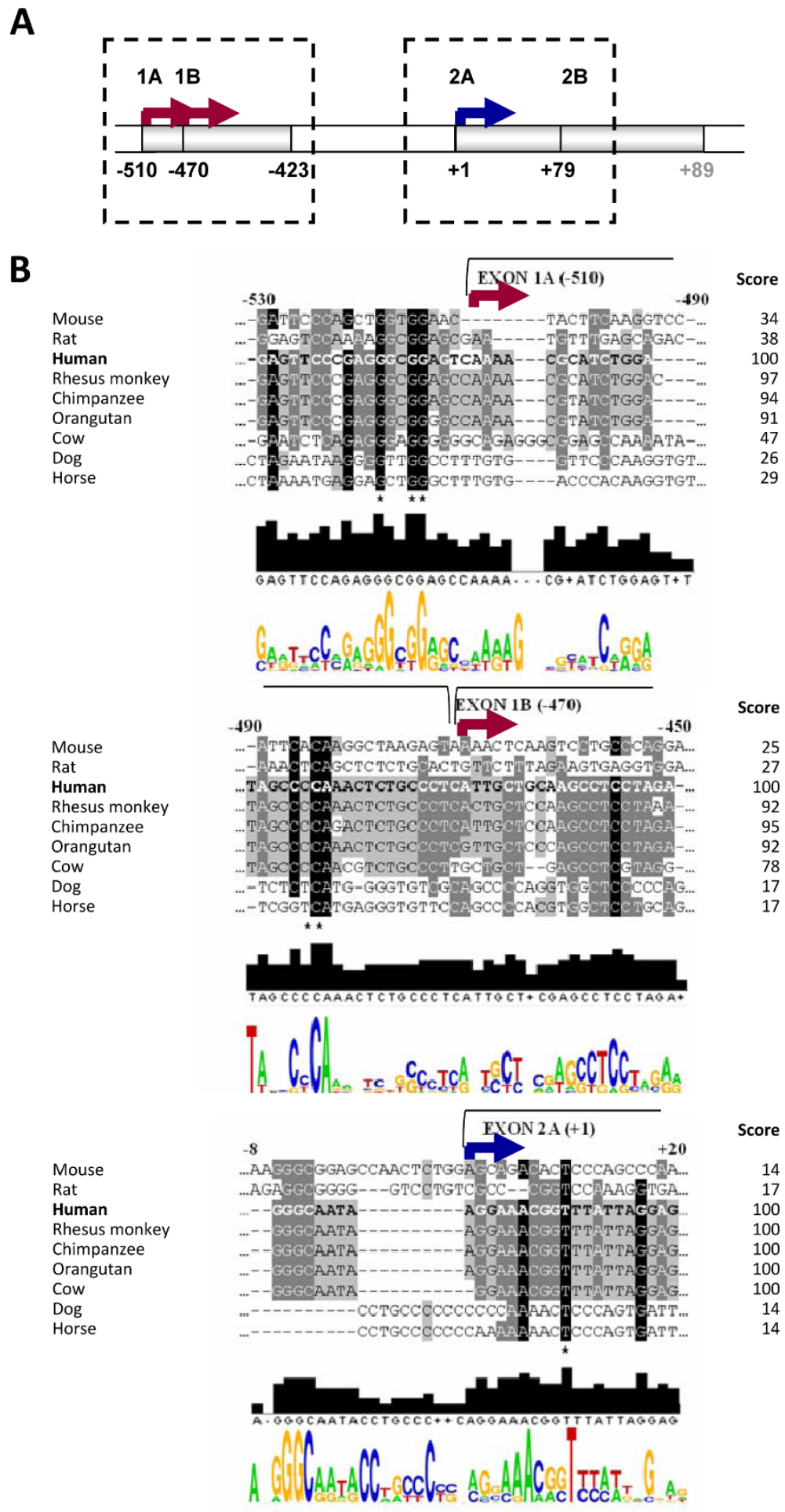

Figure 3 Sequence alignment of the human ACVRL1 TSSs across different mammalian species. (A) Scheme of the human sequences used for the alignment. The blue arrow indicates the previously described TSS $(+1)$. The red arrows indicate the new TSSs identified in Figure 1. (B) Sequences from chimpanzee, orangutan, rhesus monkey, cow, dog, horse, mouse and rat were compared with the human $-1,035 /+210$ sequence. Sequences were obtained from NCBI-GenBank and EMBL databases (see Methods). Asterisks indicate the totally conserved residues across species. Alignments of the putative regulatory sites are shown with a colored background based on the identity between species. The degree of homology is: black > dark grey > light grey. Score means percentage of identity with the human sequence. Brackets indicate exonic sequences. 


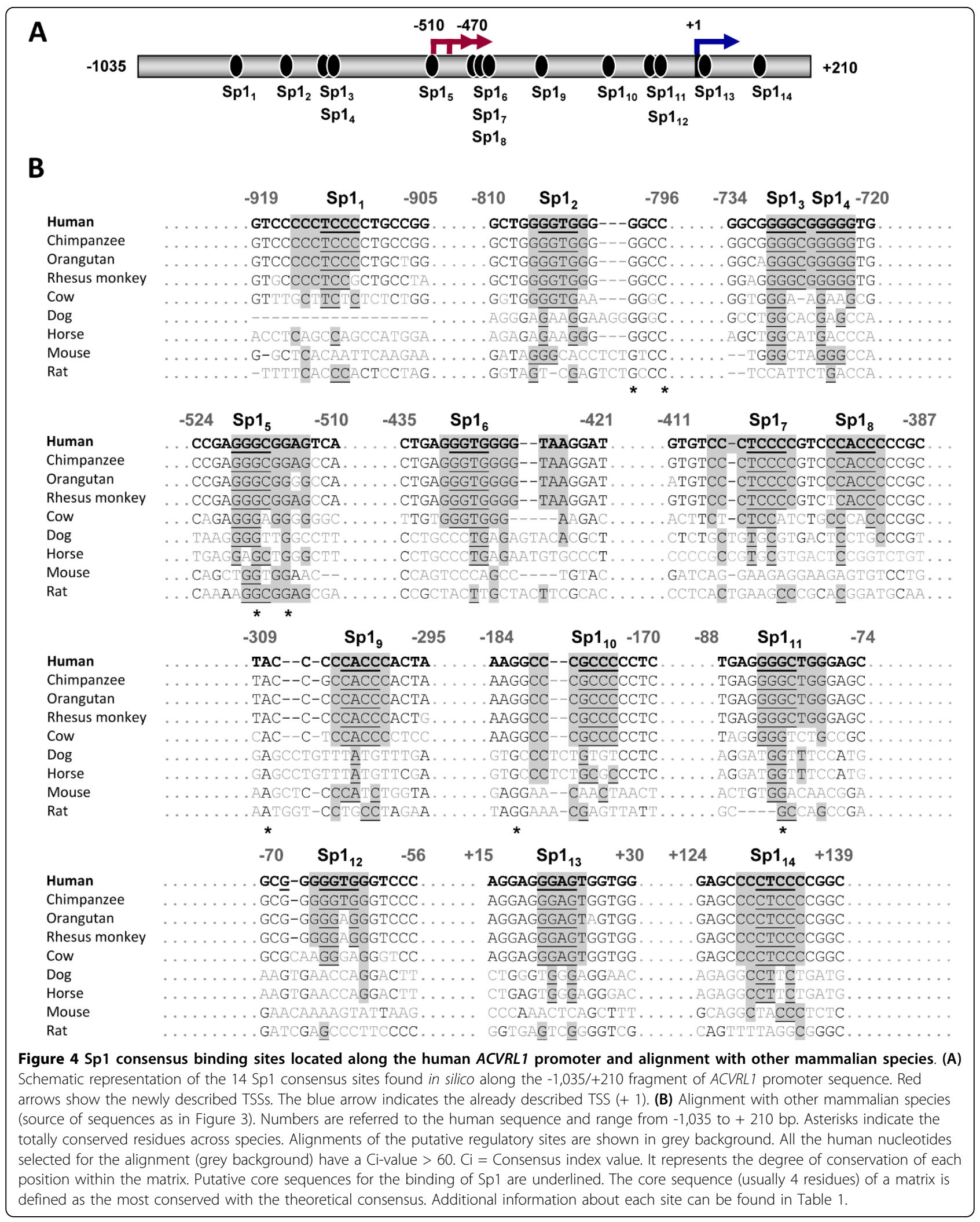




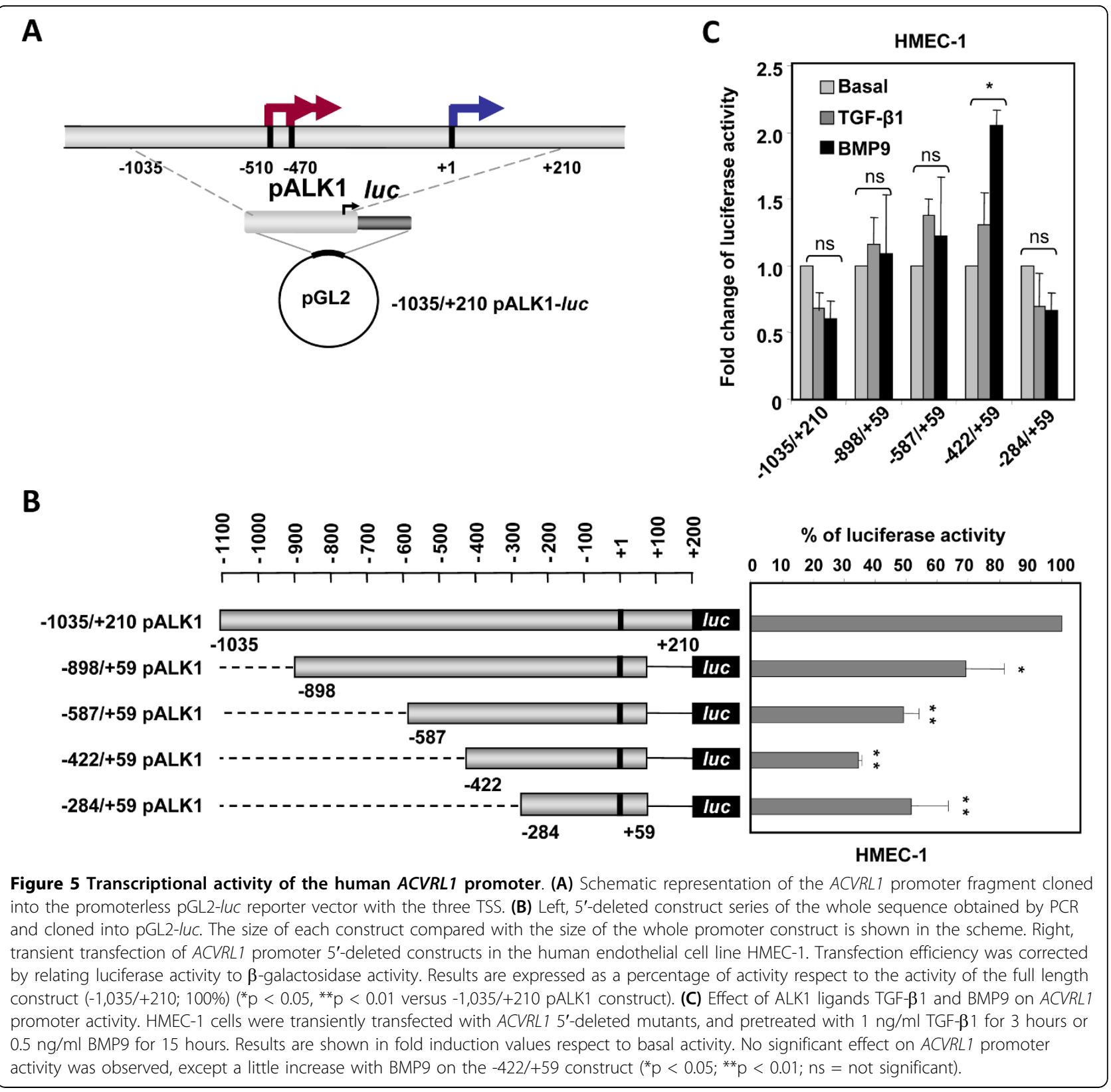

is likely due to the endogenous expression of $\mathrm{Sp} 1$ in HEK293T cells. Thus, small amounts of Sp1 expression are sufficient to saturate all the $\mathrm{Sp} 1$ sites in the ACVRL1 promoter, leading to the maximum transcriptional activity that the promoter can achieve.

The next step was to dissect the Sp1 transcription induction in the different promoter constructs. S2 cells were co-transfected with Sp1 and with the different $5^{\prime}$ deleted constructs of the reporter pALK1 (Figure 6B). All constructs showed low basal transcriptional activities, which have been referred to value 1 and the stimulation is expressed as fold induction. When as little as $25 \mathrm{ng}$ of the Sp1 expression vector was transfected, the activity levels of each construct were remarkably stimulated (between 26- to 158 -fold induction values). It is worth mentioning that the maximum activity reached by the $-422 /+59$ construct did not parallel its relatively low activity in endothelial cells, which have basal Sp1 levels (Figure 5B). This discrepancy is probably explained by the different background of transcription factors present in Drosophila embryonic versus human HMEC-1 cells.

To further assess the relevance of Sp1 in ACVRL1 transcription, Sp1 expression was abolished in mammalian Sp1-expressing cells. Thus, HEK293T cells transfected with siRNA targeted to Sp1 led to a marked decrease (more than 50\%) of the ACVRL1 


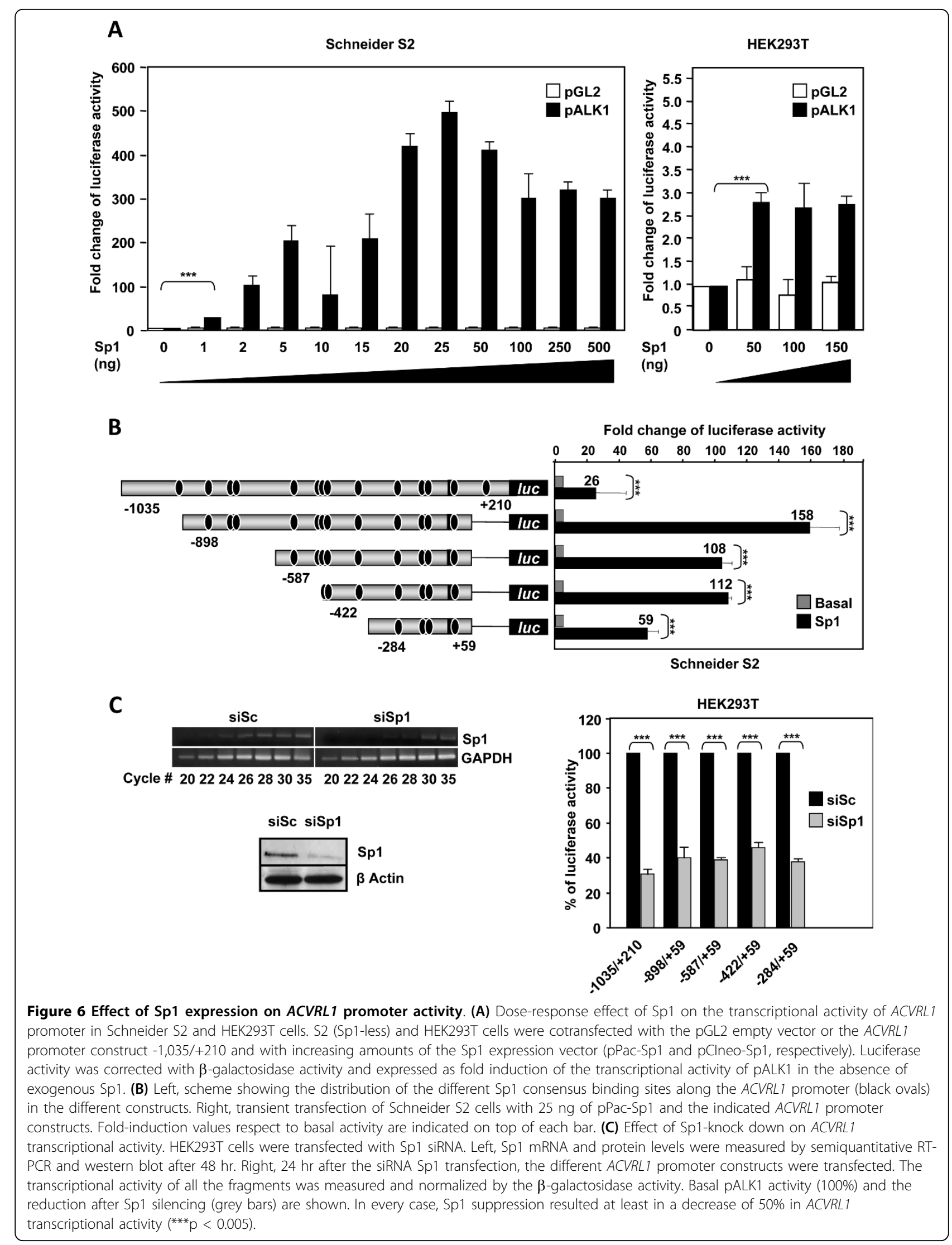


transcriptional activity in all the constructs (Figure 6C). These data confirm that $\mathrm{Sp} 1$ is essential for the transcriptional activity of the ACVRL1 promoter.

\section{Chromatin immunoprecipitation of endogenous Sp1 bound to ACVRL1 promoter in HUVECs}

Because Sp1 is clearly able to stimulate the basal transcription of ACVRL1 in vitro, it was of interest to assess whether the physical binding of Sp1 to the ACVRL1 promoter occurs within the endothelial cells. For this purpose, a chromatin immunoprecipitation (ChIP) of endogenous $\mathrm{Sp} 1$ was performed in HUVECs. Four different regions were screened, spanning the whole $-1,035 /+210$ bp sequence of $A C V R L 1$ promoter (Figure 7A). PCR amplification yielded positive bands along the four fragments, suggesting that $\mathrm{Sp} 1$ is normally bound to all these fragments of the ACVRL1 promoter and, consequently, most of the putative theoretical binding sites are functionally active (Figure 7B). Densitometric analysis of the PCR enriched fragments revealed that all of them showed a clear Sp1 binding above background, yielding the strongest signal the fragment $-510 /-260$ (Figure 7D). As a negative control, the erythropoietin $(E P O)$ promoter, containing a $\mathrm{Sp} 1$ motif that recruits Sp1 only under hypoxic conditions [36], was used. In fact, the ChIP experiment demonstrated that Sp1 did not bind to this Sp1 site in the EPO promoter, confirming the specificity of the assay (Figure $7 \mathrm{C}$ ).

Considering that the smallest fragment $(-284 /+59)$ maintains the majority of the promoter activity respect to the whole construct (Figure 5B), the nearest Sp1 sites upstream of the TSS $(+1)$, appear to be critical for the $A C V R L 1$ basal transcription. Interestingly, the $-89 /-56$ fragment encompasses two adjacent $\mathrm{Sp} 1$ consensus elements, flanking a putative KLF6 binding site (Figure 7E). This fragment was selected as a probe for EMSA studies. A mobility shift appeared when nuclear extracts from HeLa cells (Sp1 rich) were incubated with the labelled probe and this binding was effectively competed with 100-fold excess of cold probe (Figure 7F). A supershift was observed in the presence of an antibody specific for $\mathrm{Sp} 1$ whereas anti-Sp3 or anti-NF $\kappa \mathrm{B}$ antibodies did not affect the mobility shift, demonstrating the specificity for Sp1. The slight decrease of the retarded band in the presence of anti-Sp3 may be explained by the fact that Sp1 and Sp3 often bind to the same sites. Furthermore, single mutations of either $\mathrm{Sp} 1$ sites yielded retarded bands of a weaker intensity, suggesting that both sites are binding $\mathrm{Sp} 1$ independently. As negative controls, two Sp1 unrelated sequences, -823/-795 (containing consensus binding sites for other transcription factors, none of them homologous to Sp1 motifs) and AATT (double stranded poly dA-poly $\mathrm{dT}$ ) were unable to compete with the probe, although with the AATT probe a slight non-specific reduction of the signal was observed.

\section{The methylation status modulates ALK1 expression in endothelial cells}

ACVRL1 promoter showed several GC-rich regions in the in silico analysis of the $-1,035 /+210 \mathrm{bp}$ fragment. Using CpGPlot software, two CpG islands were identified, based on their parameters: 1) CG percentage > $50 \%$; and 2) a ratio of observed-to-expected $>0.6$. These two islands comprise the regions between $-408 /-239$ and $-177 /+28$ (Figure 8A). Because these GC-rich regions are potential targets for $\mathrm{Sp} 1$ binding and methylation of cytosines in $\mathrm{CpG}$ islands is one of the main epigenetic modifications that regulate gene expression, we analyzed whether ALK1 expression could be influenced by promoter methylation. Indeed, as observed by real time PCR, when endothelial HMEC-1 and HUVEC cells were subjected to a treatment with the demethylating agent 5'-aza-2'-deoxycytidine (5-aza-dC), their ALK1 levels were upregulated up to $\sim 4,000$-fold (Figure $8 B$ ). Interestingly, HEK293T, a cell line that does not express ALK1 under normal conditions (Figure 8C), underwent $\sim 6,000$-fold activation of ALK1 expression after treatment with 5 -aza-dC (Figure $8 \mathrm{~B}$ ). Likewise, Id1, which is a specific target gene of the ALK1/TGF- $\beta 1$ signaling cascade activation, became upregulated in parallel to the increased levels of ALK1 in the three cell types (Figure $8 \mathrm{~B}, \mathrm{C})$. Moreover, the activity of the reporter $\mathrm{p}(\mathrm{BRE})_{2}$ $l u c$, specific of the ALK1 signalling pathway, was strongly enhanced upon treatment with 5 -aza-dC. Conversely, the effect of in vitro hypermethylation was assessed. ACVRL1 promoter constructs were methylated in vitro with the CpG methyltransferase M.SssI and the methylation status was checked by comparative digestion of both mock and methylated ACVRL1 promoter construct with HpaII (data not shown). Then, HEK293T cells were transfected with mock-methylated or hypermethylated promoter constructs. As shown in Figure 9A the transcriptional activity of all the ACVRL1 constructs was completely abolished upon methylation. By contrast, the activity of the prolactin promoter region driven by a TATA box was almost unaffected $(\sim 80 \%$ versus untreated), whereas the activity of Id1 promoter construct was reduced at a much lower degree than the ACVRL 1 construct ( $11 \%$ versus $2 \%$ of pALK1 in HEK293T; 29\% versus 9\% of pALK1 in HMEC-1) (Figure 9B). To assess whether the methylation status of the Sp1 motifs affected the Sp1 binding, the -89/-56 radiolabelled probe was competed with cold unmethylated and methylated probes. As shown in Figure 9C, the unmethylated probe clearly inhibited the Sp1 band, whereas the methylated probe was unable to compete. 


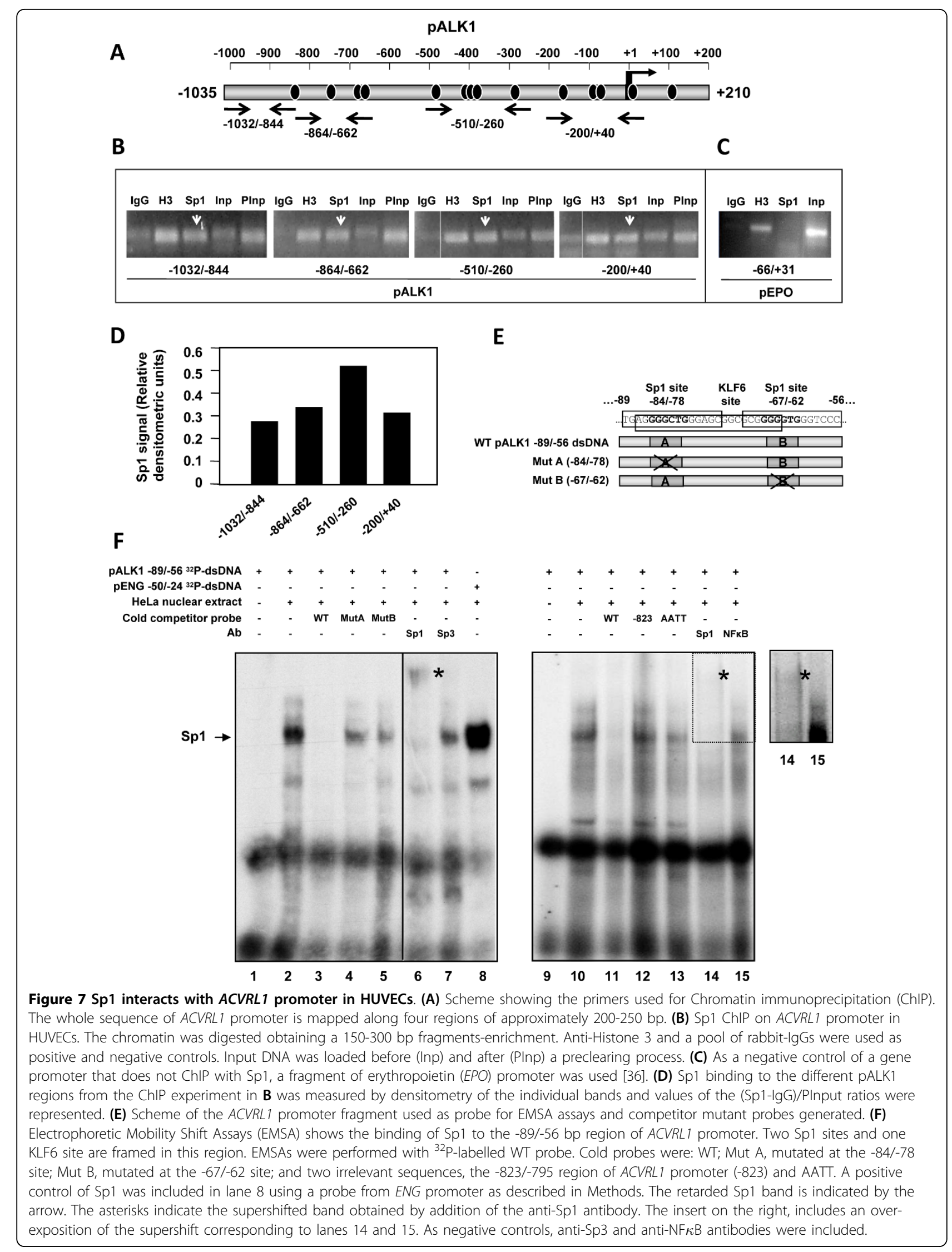


A
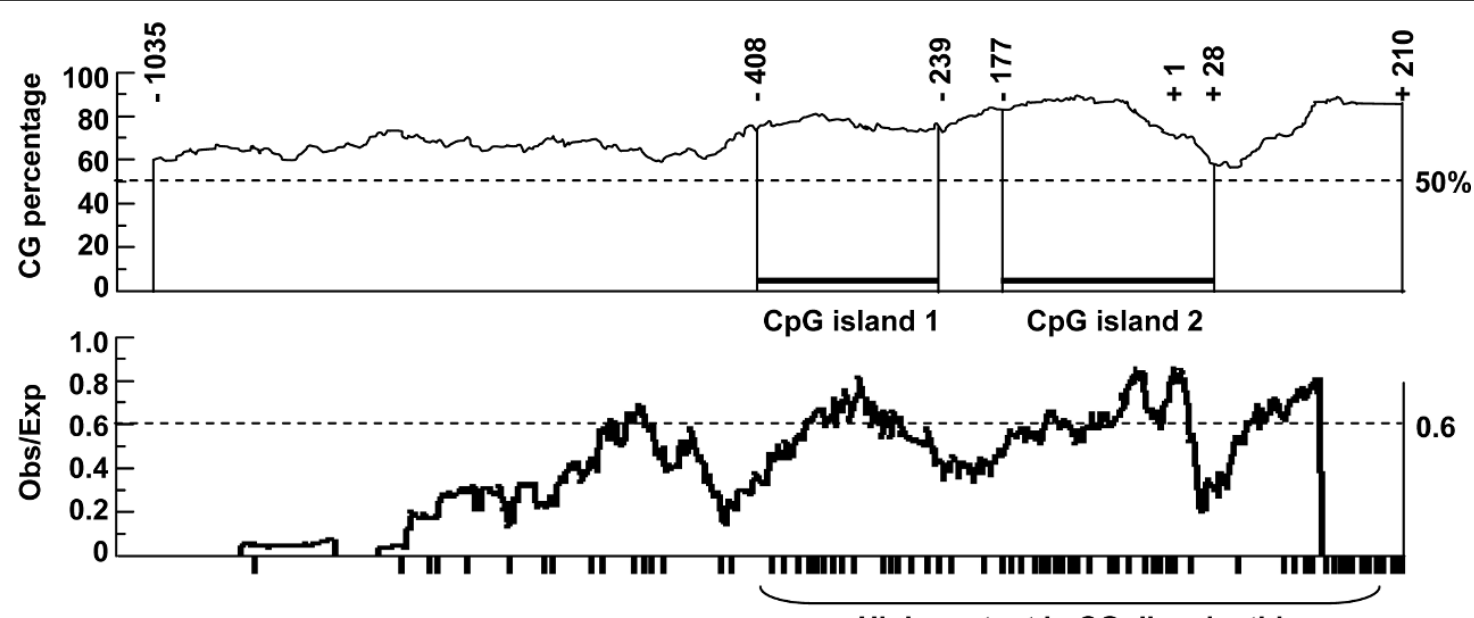

High content in CG dinucleotides

B
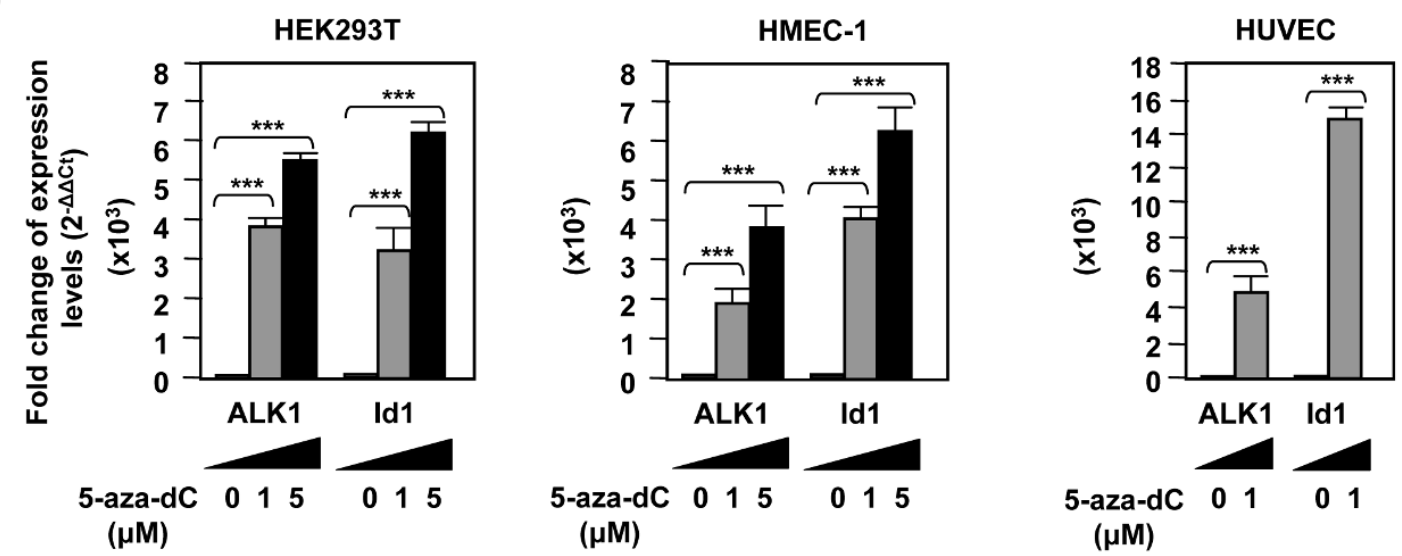

C
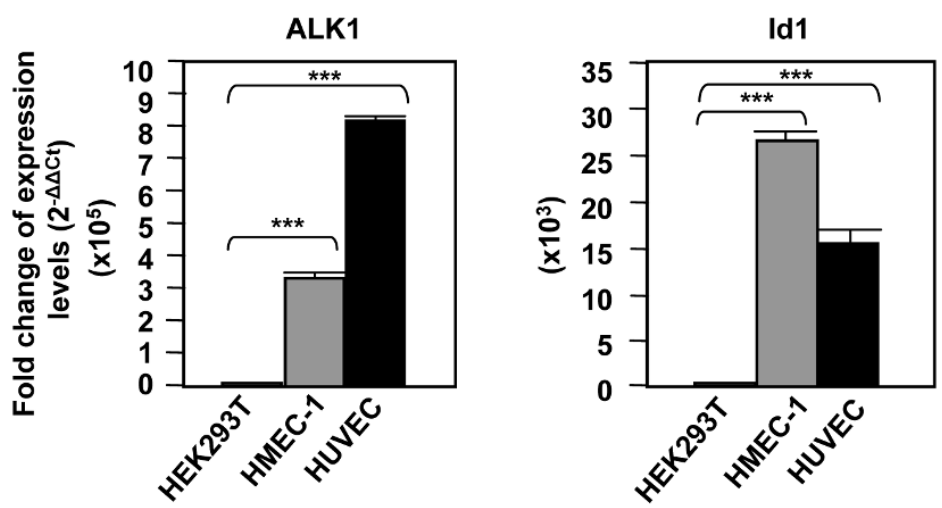

D

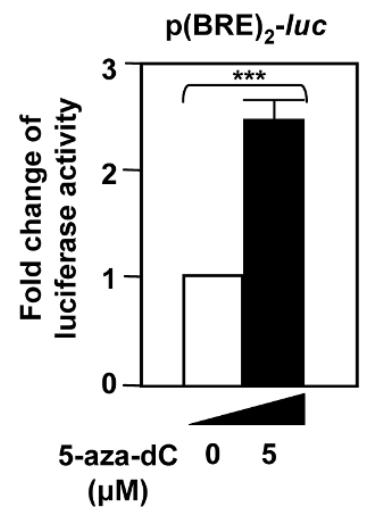

Figure 8 Two CpG islands are present in the ACVRL1 promoter and treatment with the demethylating agent 5'-aza-2'-deoxycytidine increases ALK1 expression in endothelial cells. (A) Schematic representation of the ACVRL1 promoter comprising the region $-1,035$ to +210 bp. CG sites are depicted by black bars. Two CpG islands near the transcriptional start site were detected using CpGplot software tool. CG content is shown as percentage the total number of $\mathrm{G}+\mathrm{C}$ (top), and by the methylation-susceptible CG pairs, represented by the observedversus-expected index (bottom). (B, C) ALK1 and Id1 transcript levels from endothelial (HUVEC, HMEC-1) versus non endothelial (HEK293T) cells prior and after treatment with the demethylating agent 5-aza-dC. Id1 mRNA levels were measured as a target gene of ALK1 signalling. (B) Cells were treated with $1 \mu \mathrm{M}$ or $5 \mu \mathrm{M}$ 5-aza-dC for one week. Treatment with $5 \mu \mathrm{M}$ was cytotoxic in HUVEC. RNA was extracted and mRNA levels were measured by real time RT-PCR. Results are shown as the fold change respect to basal expression ( $2^{-\Delta \Delta C t}$ ). (C) Basal ALK1 and Id1 levels show the differences between ALK1 expression in endothelial cells HMEC-1 and HUVEC versus the HEK293T cells. (D) Effect of the demethylating agent 5-aza-dC on the ALK1 pathway specific reporter, p(BRE) ${ }_{2}-\mathrm{luc}$, in HMEC-1 cells. Results are shown as fold change of expression levels or luciferase activity $\left({ }^{* * *} p<0.005\right)$. 


\section{A}

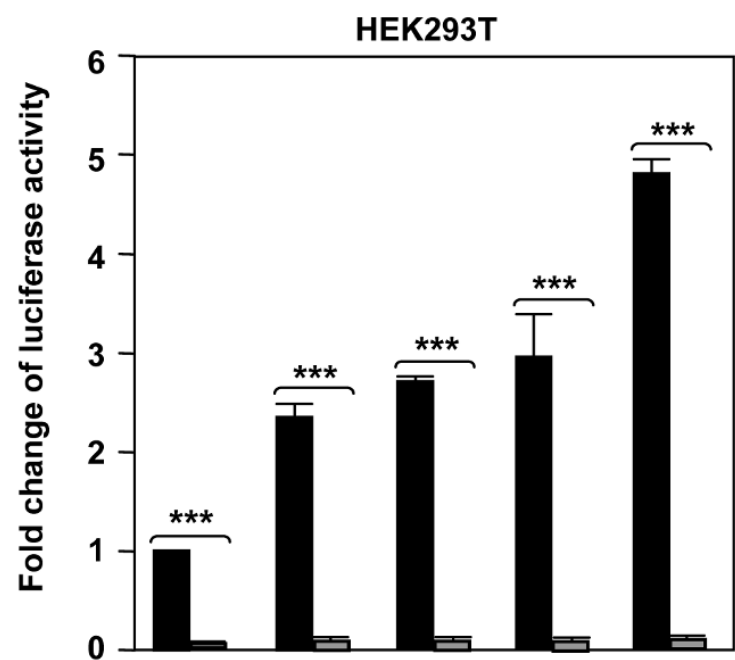

In vitro methylation

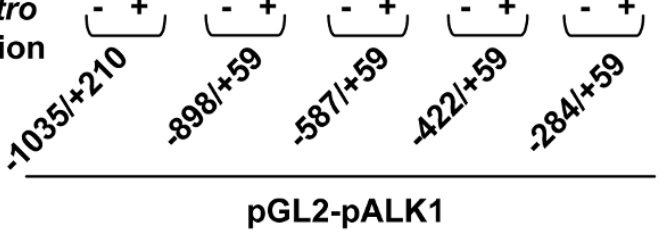

B

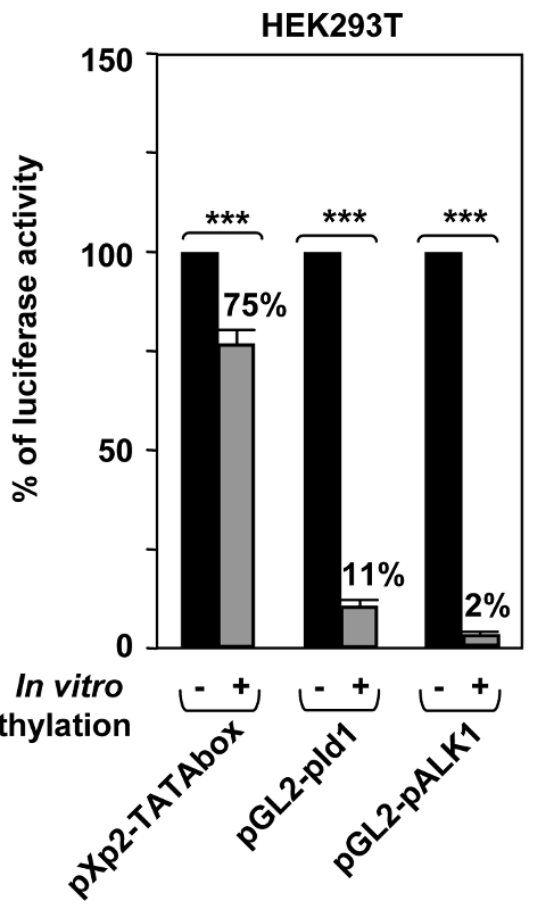

C
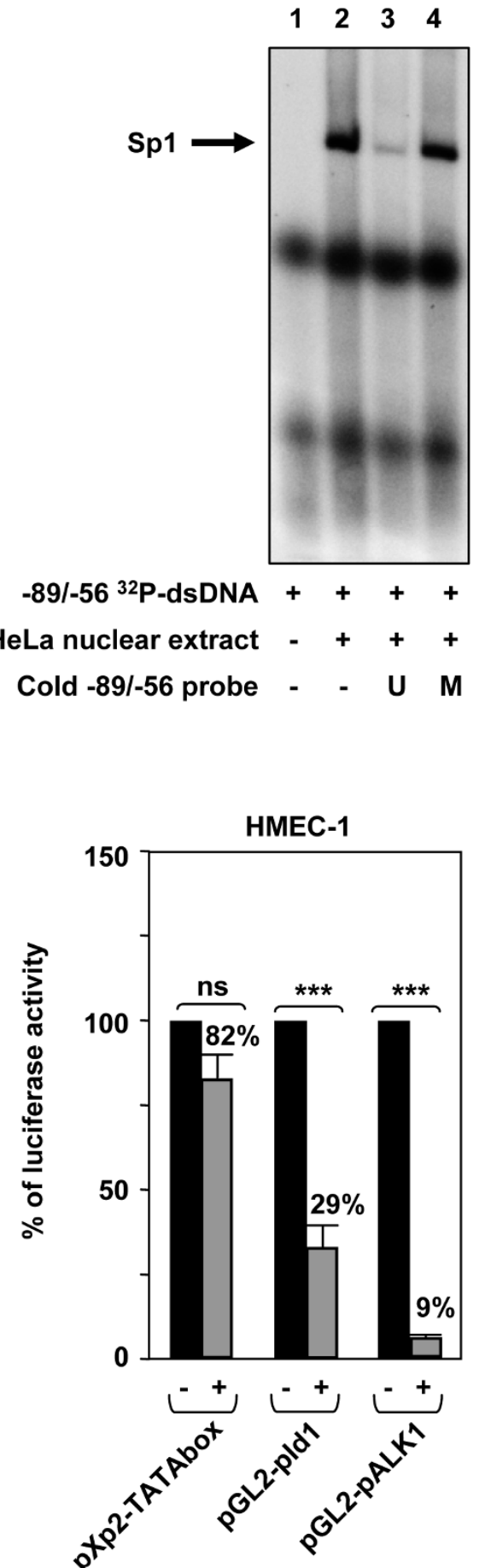

Figure 9 Effect of in vitro methylation on the ACVRL1 promoter activity. (A, B) Different reporter constructs were subjected to treatment with M.Sssl in the presence or absence of the substrate S-adenosyl-methyonine. Both mock-methylated and methylated constructs were transfected in HEK293T and HMEC-1 cells and the luciferase activity was measured. (**p < 0.005; ns = not significant). (A) Analysis of the different pALK1 reporter constructs. Results are shown as fold changes of luciferase activity (B) Analysis of a TATAbox minimal promoter (see Methods), the Id 1 promoter construct and the $-1035 /+210$ pALK1 reporter construct. Activities of untreated samples were given the arbitrary value of $100 \%$ and the activity of treated samples is indicated as percentage. (C) Electrophoretic mobility shift assay (EMSA) of the radiolabelled -89/-56 probe containing two Sp1 functional sites. Competition was carried out using the unmethylated (U) or the in vitro methylated (M) probe, as indicated. 
These results suggest that the methylation status of the promoter strongly modulates ALK1 expression.

\section{Discussion}

The characterization of the 5'-proximal ACVRL1 promoter fragment has allowed us to identify two new transcripts, upstream of the previously published TSS. Those transcripts include an upstream elusive exon, meaning that $A C V R L 1$ may have several TSSs. Thus, ACVRL1, rather than showing a unique transcription start site, contains different regions with a variable probability of transcription initiation, a characteristic of TATA-less genes. Interestingly, this is a common feature shared between $A C V R L 1$ and ENG. It is tempting to speculate that the existence of different points of transcription initiation may allow a more flexible regulation depending on the tissue, or the developmental stage of the cell. In certain tissues like placenta, two main types of transcripts have been reported [21,22]. In HUVECs we have found two new transcripts that do not affect the open reading frame of the encoded protein. In the new transcripts, the first transcribed exon is not translated, and therefore no changes in the predicted protein sequence are observed. This observation emphasizes the importance of postranscriptional as well as transcriptional regulation mechanisms in the case of ACVRL1. The existence of different transcribed untranslated regions suggests regulation of ALK1 expression that involves the transcriptional rate, mRNA stability or interaction with other RNAs or RNA binding proteins. This feature is compatible with the high degree of conservation found among primates. Also, all the ACVRL1 transcripts described in human, orangutan, rhesus monkey and chimpanzee, seem to begin with an untranslated first exon. Thus, ACVRL1 may have important motifs for transcriptional regulation in the first exon. These motifs are included in the promoter fragment that has been subjected to study in this work.

Analysis of the basal promoter activity using luciferase-reporter experiments in a series of 5 -deleted mutant constructs revealed the existence of positive and negative regulatory elements. Interestingly, the shortest construct $(-284 /+59)$ displays a similar activity to that of the whole fragment $(-1,035 /+210)$. This finding suggests the existence of critical transcription factors binding to this area in order to enhance the transcriptional activity of the TATA-less ACVRL1 promoter. In agreement with this view, a high degree of conservation around the +1 TSS among different species was found. The analysis also suggests the presence of a putative "repressor" segment between -422 and -284 positions, which inhibits $A C V R L 1$ transcription. Interestingly, a similar region is found in the ENG promoter further upstream [30], something that deserves further investigation.
The in silico study of the ACVRL1 promoter sequence showed several important putative consensus elements, also found in ENG promoter [30,32] that could be critical for the coordinated transcriptional regulation of these genes. This finding suggests that genes involved in the same functional pathway are controlled in a similar manner. Thus, ACVRL1 may be regulated by some of the transcription factors also controlling ENG expression. In this sense, because ENG is regulated by HIF-1, Smad, KLF6, Ets, or Sp1 [31-33,37], it will be of interest to assess their functional implications in ACVRL1. Of note, three sequences matching HIF-1 $\alpha$ motifs were found at positions $-808 /-792,-286 /-274$ and $-236 /-219$ bp. The possibility that ACVRL1 could respond to HIF$1 \alpha$ is supported because hypoxia promotes angiogenesis, a process that is regulated by ALK1. In addition, multiple KLF consensus elements are dispersed along the $A C V R L 1$ promoter region. Interestingly, this is another feature owing to the TGF- $\beta$ receptor complex genes, in which the transcription is stimulated by KLF6 as an early injury response $[18,32]$. On the other hand, the presence of multiple Ets sites, suggests regulation by MAPKs (Mitogen Activated Protein Kinases) [38]. In this sense, in the functionally related gene, ENG, an Ets functional site was described in the proximal promoter [30]. We also note that the presence of AP1 and NF $\kappa \mathrm{B}$ sites suggests that $A C V R L 1$ could be a target for the inflammatory response. In contrast to the ENG proximal promoter that contains abundant SBE motifs, only a single putative SBE was found at -270 bp position of the $A C V R L 1$ promoter. Also, at variance with ENG, the $A C V R L 1$ promoter does not contain any TGF- $\beta$-responsive element (SBE) in the neighbourhood of Sp1 sites. Moreover, the TGF- $\beta$ receptor type I (ALK5) gene (TGFBRI), contains a SBE within the proximal promoter and both ENG and TGFBRI mRNA expression is upregulated by TGF- $\beta[30,39]$. Thus, the proximal region of $E N G$ is regulated by TGF- $\beta$ through Smads, and a direct cooperation Smad/Sp1 giving rise to an important increase in transcription [31]. Consistent with the lack of proximal responsive TGF- $\beta$ elements within the $A C V R L 1$ promoter, we found that its promoter activity was not regulated by TGF- $\beta 1$ or BMP9. This does not exclude the possibility of a transcriptional regulation by TGF- $\beta$ from SBEs placed further upstream, or by an indirect postranscriptional control through modifications of the receptor, which allow or prevent ALK1 to activate signalling through Smads. Addressing the functionality of all these transcription factors in the ACVRL1 promoter deserves an independent investigation. The present work has focused on the functional role of Sp1 on ACVRL1 basal transcription.

The most striking feature revealed by the in silico analysis of ACVRL1 is the presence of multiple Sp1 sites 
next to the different TSSs. Sp1 has been widely described as a general transcription factor involved in transcriptional basal mechanisms of gene promoters that lack TATA and CAAT core boxes. This is a shared characteristic among different housekeeping genes [40]. Interestingly, this is also the case of several TGF- $\beta$ receptors such as ENG and TGFBR1, whose transcription is driven by $\mathrm{Sp} 1[30,31]$. Further support for the involvement of Sp1 was obtained from the highly conserved alignment of the $\mathrm{Sp} 1$ consensus sites along the $A C V R L 1$ promoter among several species that suggests a conserved biological relevance of $\mathrm{Sp} 1$ in the transcription of ACVRL1.

We have demonstrated here that $\mathrm{Sp} 1$ is a key factor necessary for the basal activation of ACVRL1 transcription. This effect has been observed both by $\mathrm{Sp} 1$ overexpression in cells lacking endogenous $\mathrm{Sp} 1$, and by interfering endogenous $\mathrm{Sp} 1$ with siRNA in mammalian cells. The large number of $\mathrm{Sp} 1$ consensus sites found in the $A C V R L 1$ promoter, and the finding that small amounts of $\mathrm{Sp} 1$ can saturate its transcription, points out to a critical dependence on Sp1, and a fine tuning of ACVRL1 transcription by Sp1. Furthermore, a ChIP analysis in HUVECs after chromatin crosslinking revealed that $\mathrm{Sp} 1$ binds in vivo to the different consensus motifs along the whole proximal $A C V R L 1$ promoter sequence studied $(-1,032 /+40)$. Thus, in endothelial primary cells growing in a rich medium, $\mathrm{Sp} 1$ may be binding to most of the specific motifs within the ACVRL1 promoter region. It can be speculated that the $\mathrm{Sp} 1$ binding to the starting transcriptional machinery complex is needed to ensure a transcription driven by Sp1 using different TSSs.

Because the closest region to the +1 TSS is framed by the construction $-284 /+59$ pALK1 and its transcriptional levels are similar to those of the $-1,035 /+210$ pALK1 vector, probably the functional involvement of $\mathrm{Sp} 1 \mathrm{for}$ ACVRL1 transcription initiation is critical in this region. When searching the $\mathrm{Sp} 1$ consensus sites within this region, an interesting double site in the $-89 /-56$ region was found. This Sp1 rich fragment contains two putative Sp1 consensus sites (-84/-78 and -67/-62) and, as shown in EMSA experiments, both sites are functional in binding to Sp1.

One key factor that modulates the Sp1 transcription dependency is the degree of methylation of the CpG islands contained within the $\mathrm{Sp} 1$ consensus elements. This is consistent with the observation that DNA methylation may interfere with the binding of $\mathrm{Sp} 1$ to DNA [41]. In fact, we found that methylation of the $-89 /-56$ Sp1 probe of $A C V R L 1$ rendered this consensus motif inactive for Sp1 binding. It is well established that DNA methylation of $\mathrm{CpG}$ islands is an important mechanism for transcriptional regulation of multiple genes in mammals [42]. However, most of these findings are related to proto-oncogenes, meaning that the hypermethylation is a protective mechanism for controlling the transcriptional switch to oncogenes. On the other hand, hypomethylation of tumor suppressor genes is a control mechanism for assuring its transcriptional rate. However, there are not many reports of genes non-related to cancer that could be controlled by their methylation state. For example, demethylation of certain promoters is involved in the return of somatic cells to previous undifferentiated stages of their cell lineage, and in the reprogramming to a new differentiating pathway in response to certain stimuli [43]. In some genes, cytosine methylation of Sp1 sites has been reported as a marker of organ-specific expression and as a specific regulator of the expression [44]. That could be the case of $A C V R L 1$, in which demethylation of DNA in endothelial cells leads to a marked increase of ACVRL1 transcription, potentially because demethylation of $\mathrm{CpG}$ islands within the Sp1 sites is involved in ACVRL1 basal transcription.

ALK1 expression has been reported in several cell types, but its major roles are related to its predominant expression in endothelium. The ALK1 specific presence at the endothelial cell surface is tightly involved in the regulation of the TGF- $\beta$ signalling pathway in balance with other type I TGF- $\beta$ receptors [35]. The endothelial specific expression may be explained by the presence within the proximal promoter region of $A C V R L 1$ of consensus motifs for transcription factors (Ets, NF $\kappa \mathrm{B}$, Sp1 and KLFs) also shared by other endothelial specific genes such as ENG, PECAM1 (Platelet Endothelial Cell Adhesion Molecule 1), VEGFR2 (Vascular Endothelial Growth Factor Receptor 2), CDH5 (Cadherin 5), eNOS/ NOS-3, or TIE2 (Tyrosine kinase with Immunoglobulinlike and EGF-like domains 2) [30,31,45-49]. In addition, the involvement of distal regulatory regions in the human ACVRL1, such as the one described in mouse that confers arterial endothelium-specificity, can not be excluded [50]. Whether the degree of ACVRL1 methylation correlates with the endothelial specific expression of ALK1, remains to be explored.

\section{Conclusions}

Novel ACVRL1 transcripts have been identified, the $A C V R L 1$ promoter has been characterized and its regulation by $\mathrm{Sp} 1$ has been demonstrated. Furthermore, a close dependence between ACVRL1 expression and the CG methylation degree was found. Future experiments to identify other trans-acting or trans-repressing factors in ACVRL1 regulation remain to be addressed.

\section{Methods}

5'Rapid Amplification of CDNA Ends (5'RACE)

RNA from human umbilical vein endothelial cells (HUVEC) was extracted and purified with RNeasy 
(Qiagen, Hilden, Germany) following the manufacturer's protocol. 5' RNA-Ligase-Mediated rapid amplification of cDNA ends (RLM-RACE) was carried out according to the manufacturer's instructions (Ambion Inc., Austin, TX, USA). Prior to the reverse transcription-polymerase chain reaction (RT-PCR), total RNA was treated with calf intestinal phosphatase (CIP) to remove the 5'phosphate from all RNA species except intact mRNA bearing the the 5' CAP structure. Then, tobacco acid pyrophosphatase (TAP) was used to remove the cap structure from mRNA leaving a $5^{\prime}$-phosphate exposed and available for the subsequent ligation. The use of a CIP/TAPpre-treatment increases 5'RACE selectivity by avoiding the presence of false PCR amplification products, by selecting the complete mRNAs from the total RNA population. Next, a synthetic RNA oligonucleotide was added and ligated to the CIP/TAP treated RNA and the chimeric RNA was reverse transcribed using random primers. RT-PCR was performed with the avian-myeloblastosis virus (AMV) reverse transcriptase (RT) kit (Roche Diagnostics, Mannheim, Germany). The resulting CDNA was used as a template for two nested PCR. PCR was performed using different forward oligonucleotides complementary to the 5'RACE adapter and two reverse $A C V R L 1$-specific primers, outer and inner, respectively. The sequences of the primers are indicated in Table 2. PCR was performed using an annealing temperature of $58^{\circ} \mathrm{C}$, and with HotMaster Taq polymerase (Eppendorf, Westbury, NY, USA).

\section{Real time PCR}

For quantitative analysis, total RNA was isolated from cells using the RNeasy kit (Qiagen) and was reversetranscribed using AMV reverse transcriptase (Roche Diagnostics). The resultant cDNA was used as a template for real time PCR performed with the primers shown in Table 2 using the iQ SyBR-Green Supermix (BioRad, Hercules, CA, USA). Amplicons were detected using an iQ5 real time detection system (BioRad). Transcript levels were normalized to $18 \mathrm{~S}$ levels. Triplicates of each experiment were performed.

\section{Cloning of the ACVRL1 promoter fragment}

A genomic $A C V R L 1$ fragment corresponding to the 5'proximal region upstream the $(+1)$ TSS was cloned into $\mathrm{SacI} / \mathrm{XhoI}$ sites of the reporter vector pGL2-luc containing the promoterless firefly luciferase gene (Promega, Madison, WI, USA). This construct comprises 1,244 bp and extends from the position $50,586,434$ to $50,587,679$ of the contig (GenBank: NC_000012.10. Reference Assembly). The construct was checked by sequencing with pGL primers 1 (Forward) and 2 (Reverse) (Promega) and the resulting sequence was identical to that of the GenBank.

\section{Alignment among different species and in silico analysis of the ACVRL1 promoter}

The human $A C V R L 1$ promoter sequence was compared with the orthologous promoters in a set of animal species. The sequences to be compared were chosen by identifying the theoretical +1 TSS and then aligning the human $-1,035 /+210$ bp region. The accession numbers of the different contigs and the sequences used are: Mus musculus [GenBank:NT_039621] 62,243,937 $62,245,182$, plus strand; Rattus norvegicus [GenBank: NW_047784] 6,594,766 - 6,596,011, plus strand; Bos taurus [GenBank:NW_001495018] 123,378 - 122,133, minus strand; Canis familiaris [GenBank:NW_76284] 3,087,613-3,086,368, minus strand; Pan troglodytes [GenBank:NC_006479] 2,296,228 - 2,294,983, minus strand; Pongo pygmaeus [EMBL:413.52] 51,613,841 - 51,615,086, plus strand; and Macaca mulatta [GenBank: NW_001096621] 1,104,215 - 1,105,460, plus strand. For the in silico analysis of the putative response elements, we used the Genomatix MatInspector software tool: http://www.genomatix.de/products/MatInspector. The multiple sequence alignment was performed with the ClustalW2 software http://www.ebi.ac.uk/Tools/clustalw2, which generates a similarity score (values from 1 to 100) and provides a consensus proposal. Weblogo tool http://weblogo.berkeley.edu/logo.cgi was very useful to make the graphic representation of these consensus regions.

\section{Generation of 5 'deleted fragments of the human ACVRL1 promoter cloned into pGL2}

Four different constructs with serial deletions of the $A C V R L 1$ promoter were generated by PCR amplification, using primers designed to generate fragments with differences of $\sim 150$ bp between each other. All the sequences of the cloning primers are given in Table 2 . Forward primers were at positions: $-898 /-880,-587 /-$ $569,-422 /-404$ and $-281 /-263$. In all cases, the reverse primer was at positions: $+42 /+59$. The amplified fragments were: pALK1 -898/+59 (957 bp); pALK1 -587/ +59 (646 bp); pALK1 -422/+59 (481 bp) and pALK1 $-284 /+59$ (343 bp). The resulting products were purified and cloned into pCR2.1-TOPO-TA vector (Invitrogen, Carlsbad, CA, USA). Then, they were digested using $S a c$ I and XhoI flanking restriction sites, inserted in pGL2-luc and checked by sequencing with commercial pGL primers 1 and 2 as described above.

\section{Cell culture}

Drosophila Schneider S2 cells were grown in Drosophila-enriched Schneider's (DES) insect medium (Sigma Aldrich, St Louis, MO, USA) supplemented with 10\% fetal bovine serum (FBS), $0.1 \mu \mathrm{g} / \mu \mathrm{l}$ gentamicin and 2 $\mathrm{mM}$ L-glutamine. The human microvasculature 
endothelial cell line HMEC-1 was grown on $0.2 \%$ gelatin (Sigma Aldrich) pre-coated plates in MCDB-131 medium (Gibco, Paisley, UK) supplemented with 10\% FBS, 2 $\mathrm{mM}$ L-glutamine, $1 \mathrm{ng} / \mathrm{mL}$ Epidermal Growth Factor (EGF) and $1 \mu \mathrm{g} / \mathrm{ml}$ hydrocortisone. Primary HUVEC were grown in EBM2 medium supplemented with EGM2 (Lonza, Walkersville, MD, USA) and containing 10\% FBS. Human epithelial embryonic kidney HEK293T cells were cultured in Dulbecco's modified Eagle's medium (DMEM) with 10\% FBS. All the media were supplemented with both $100 \mathrm{U} / \mathrm{ml}$ penicillin-streptomycin.

\section{DNA transfections and luciferase assays}

Transfections of HEK293T, HMEC-1 and Schneider S2 cells were performed using the Superfect Reagent (Qiagen) following the commercial instructions. When required, transfected cells were treated for 3 hours with $1 \mathrm{ng} / \mathrm{ml}$ of TGF- $\beta 1$ (R\&D Systems, Minneapolis, MN, USA) or 16 hours with $0.5 \mathrm{ng} / \mathrm{ml}$ of BMP9 (R\&D Systems) in the presence of $2 \%$ FBS. The expression vector $\mathrm{pCIneo-Sp1}$ was used to transfect mammalian cells, whereas pPac-Sp1 was used to transfect Drosophila Schneider S2. The corresponding empty vectors were used as controls. Forty eight hours after transfection with the pGL2 reporter vectors, cells were harvested and the luciferase activities were determined in a TD-20/20 luminometer (Promega). In all cases, the pCMV- $\beta$ Gal vector was included in the transfections and its transcriptional activity was measured as an internal control. After normalization, the activity of the reporter constructs was referred to the basal activity as fold induction or as percentages with respect to controls.

\section{Sp1 knock down}

The human Sp1 small interfering ribonucleic acid (siRNA) was obtained from Santa Cruz Biotechnology (sc-29487, Santa Cruz, CA, USA). HEK293T cells were transfected with 5 pmoles of siRNA hSp1 or scrambled siRNA, using lipofectamine 2000 (Invitrogen, Carlsbad, CA, USA). Twenty four hours later, the pALK1 reporter construct and the Renilla normalization plasmid were transfected, using the Superfect Reagent (Qiagen). Luciferase activity was measured 24 hours after DNA transfection with the Dual Luciferase Assay System (Promega). Values were normalized to Renilla activity and values were referenced to the basal activity (100\%) in each case. Lysates from these cells at 48 hours posttransfection were analyzed by semiquantitative RT-PCR using the primers indicated in Table 2 and by western blot using the rabbit polyclonal antibody anti-Sp1 (PEP2, SC-59, Santa Cruz Biotechnology) and monoclonal mouse antibody anti- $\beta$-actin antibody AC15 (A1978, Sigma Aldrich).

\section{Chromatin immunoprecipitation (ChIP)}

ChIP was performed with ChIP-IT Express kit (Active motif, Rixensart, Belgium), following the manufacturer's instructions. Briefly, HUVEC were grown to confluence and subsequently fixed with $1 \%$ formaldehyde. Cells were scraped in the presence of PMSF (phenylmethylsulphonyl fluoride) and lysed. Nuclei were separated using a dounce homogenizer and digested with enzymatic shearing cocktail for $15 \mathrm{~min}$. One aliquot of this sheared chromatin was used as "input chromatin" and the rest was incubated with protein $G$ magnetic beads and rabbit polyclonal antibody against human Sp1 PEP2 (SC-59, Santa Cruz Biotechnology) on a rolling shaker for 4 hours at $4^{\circ} \mathrm{C}$. The positive control was incubated with rabbit polyclonal anti-human Histone 3 (ab8580, Abcam, Cambridge, MA, USA) and the negative control with serum anti-human IgG. Protein G magnetic beads bound to the immune complexes were pelleted, washed and bound proteins were eluted with the elution buffer provided with the kit. Then, the crosslinking was reversed and samples were incubated with proteinase $\mathrm{K}$ during $1 \mathrm{~h}$ at $37^{\circ} \mathrm{C}$. Primers used for PCR were selected by mapping the whole promoter sequence, separated into four regions. The first region encompasses from $-1,032$ to -844 (188 bp); the second region from -864 to -662 (202 bp); the third region from -510 to $-260(250$ bp) and the fourth region from -200 to +40 (240 bp). Sequences of the four couples of primers are indicated in Table 2. For negative and positive control PCR, primers from ChIP-IT control kit human (Cat \# 53010, Active motif) were used (data not shown). Measurement of the Sp1 binding was carried out using the following ratio of band intensities: (Sp1-IgG)/PInput.

\section{Electrophoretic mobility shift assay (EMSA)}

For the radiolabelled probe, the oligonucleotides were designed in the region -89/-56 of the ACVRL1 promoter, which includes two Sp1 consensus sites flanking a Krüppel-like factor-6 (KLF6) site. Competition experiments were carried out with five cold probes: i) $-89 /-56$ wild type (WT); ii) $-89 /-56$ with the site $-84 /-78$ mutated; iii) $-89 /-56$ with the site $-67 /-62$ mutated; iv) $-823 /-795$, containing consensus binding sites for other transcription factors, none of them homologous to Sp1 motifs; and v) AATT, an artificial competitor, double stranded poly dA-poly dT sequence, unrelated to the ACVRL1 promoter. The corresponding sequences are shown in Table 2. Probes were prepared by annealing complementary synthetic oligonucleotides followed by end labelling with $\left[\gamma^{32} \mathrm{P}\right] \mathrm{dATP}$ and T4 polynucleotide kinase. Nuclear extracts from HeLa cells were obtained from Promega (Cat \# E3521). Approximately $5 \mathrm{ng}$ $(100,000 \mathrm{cpm})$ of the respective probe was incubated with $10 \mu \mathrm{g}$ of nuclear extract and $2 \mu \mathrm{g} /$ reaction of poly 
(dI-dC) for 30 min on ice. For competition experiments, a 100-fold excess of unlabeled double-stranded oligonucleotide was added. For supershift assays, protein extract and $1 \mu \mathrm{g}$ of commercial antibody were preincubated for $60 \mathrm{~min}$ on ice prior to the addition of the remaining components of the binding reaction. Rabbit polyclonal antibodies anti-Sp1 (PEP2, SC-59), anti-Sp3 (SC-644) and anti-NF $\kappa \mathrm{B}$ (Nuclear Factor kappa-lightchain-enhancer of activated B cells) (H-119, SC-7178) were purchased from Santa Cruz Biotechnology.

Positive control binding reactions were performed with a probe designed for the ENG promoter (-50/-24 Sp1 sites), which strongly binds Sp1 [31]. Binding reactions were separated by nondenaturing $6 \%$ polyacrylamide gel electrophoresis in Tris-Borate-EDTA buffer at $4^{\circ} \mathrm{C}$. Gels were dried, and visualized by autoradiography. EMSAs were repeated at least three times with similar results.

\section{Treatment of cells with the demethylating agent 5'-aza- 2'-deoxycytidine}

CpG islands were detected using the software tool CpGplot http://www.ebi.ac.uk/Tools/emboss/cpgplot/ index.html. HUVEC, HMEC-1, and HEK293T cells were treated with 5'-aza-2'-deoxycytidine (5-aza-dC; Sigma Aldrich) at a final concentrations of $1 \mu \mathrm{M}$ or $5 \mu \mathrm{M}$ for 7 days and the medium was changed every second day, according to Butta et al., [51]. Finally, cells were lysed, total RNA was extracted with RNeasy kit (Qiagen) and the ALK1/Id1 ratio was measured by real time PCR. Primers used are shown in Table 2.

For functional experiments, after a one week of treatment with $5 \mu \mathrm{M} 5$-aza-dC, HMEC-1 cells were transfected with the specific reporter of the ALK1 pathway $\mathrm{p}(\mathrm{BRE})_{2^{-}}$ luc (BMP-responsive firefly luciferase reporter), which contains a small sequence with two copies of the regions $(-1052 /-1032) /(-1105 /-1080)$ of the Id1 promoter [52].

\section{In vitro methylation of promoters}

Constructs $-1,035 /+210,-898 /+59,-587 /+59,-422 /+59$ and $-284 /+59$ of $A C V R L 1$ promoter and the reporter plasmids of Id1 promoter (pGL2-pId1) and the rat minimal prolactin promoter region -36 to +37 surrounding a TATA box, (pXP2-TATAbox) [53] were methylated in vitro by the CpG methylase M.SssI (New England Biolabs, Ipswich, MA, USA) in the absence or presence of the substrate $\mathrm{S}$-adenosylmethionine. The methylation status was checked by digestion with the restriction enzyme HpaII, which digests the target sequence CCGG but only when it is unmethylated (data not shown). Cells were transfected with $100 \mathrm{ng}$ of the indicated mock-methylated or methylated ACVRL1 promoter reporter, $100 \mathrm{ng}$ of pCIneo-Sp1 and with Renilla reporter vector. Luciferase activity was measured after 48 hours and normalized to Renilla activity. For electrophoretic mobility shift assays, the cold probe (-89/-56) of $A C V R L 1$ promoter was subjected to in vitro methylation with the CpG methylase M.SssI prior to competition with the radiolabelled probe.

\section{Statistics}

Data were subjected to statistical analysis and results are shown as mean \pm SD. Differences in mean values were analysed using Student's t-test. In the figures, the statistically significant values are marked with asterisks

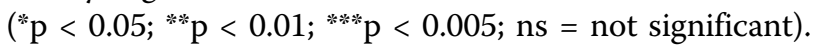

\section{Abbreviations}

ACVRL1: activin-A receptor type I-like kinase 1; ALK1: Activin receptor-Like Kinase 1; 5-aza-dC: 5'-aza-2'-deoxycytidine; BMP9: Bone Morphogenetic Protein-9; EMSA: Electrophoretic mobility shift assay; ENG: Endoglin; eNOS/ NOS-3: Endothelial Nitric Oxide Synthase; Ets: E26-Transformation-specific Transcription Factor; FBS: Fetal Bovine Serum; HHT: Hereditary Hemorrhagic Telangiectasia; HIF: Hypoxia Inducible Factor; KLF6: Krüppel-Like Factor 6; $\mathrm{NF} \kappa \mathrm{B}$ : Nuclear Factor of kappa light polypeptide gene enhancer in B-cells; 5' RACE: 5'Rapid Amplification of CDNA ends; RXR: Retinoid X Receptor; SBE: Smad Binding Element; Sp1: Specificity Protein 1; TGF- $\beta$ : Transforming Growth Factor- $\beta$; TSS: Transcriptional Start Site.

\section{Acknowledgements}

We thank Dr. Jonathan N. Berg (Clinical Genetics Ninewells Hospital and Medical School Dundee, UK) for providing the genomic DNA fragment of ACVRL 1 promoter, and Drs. Tilman Sánchez-Elsner and Nora Butta for their helpful advices. This work was supported by grants from Ministerio de Ciencia e Innovación of Spain (SAF2007-61827 to CB; SAF08-01218 to LMB, and predoctoral fellowship BES-2005-7974 to EMG-M), National Institutes of Health \& National Center for Research Resources (P20-RR-15555 and HL083151 to CPV; DK56621 and DK37340 to SLF) and Centro de Investigación Biomédica en Red de Enfermedades Raras (CIBERER). The CIBER de Enfermedades Raras is an initiative of the Instituto de Salud Carlos III (ISCIII) of Spain.

\section{Author details}

'Centro de Investigaciones Biológicas (CIB), Consejo Superior de Investigaciones Cientificas (CSIC) and Centro de Investigación Biomédica en Red de Enfermedades Raras (CIBERER), Ramiro de Maeztu 9, 28040 Madrid, Spain. ${ }^{2}$ Department of Cancer Biology and Genetics, Memorial SloanKettering Cancer Center, New York, NY, USA. ${ }^{3}$ Center for Molecular Medicine, Maine Medical Center Research Institute, Scarborough, ME, USA. ${ }^{4}$ Division of Liver Diseases. Mount Sinai School of Medicine, New York, NY, USA.

\section{Authors' contributions}

EMG-M, FJB, CB and LMB conceived, designed and coordinated the study and wrote the manuscript. All authors participated in analysis of the results. All authors have read and approved the final manuscript.

Received: 16 November 2009 Accepted: 29 June 2010 Published: 29 June 2010

\section{References}

1. Johnson DW, Berg JN, Baldwin MA, Gallione CJ, Marondel I, Yoon SJ, Stenzel TT, Speer M, Pericak-Vance MA, Diamond A, et al: Mutations in the activin receptor-like kinase 1 gene in hereditary haemorrhagic telangiectasia type 2. Nat Genet 1996, 13:189-195.

2. Su Al, Wiltshire T, Batalov S, Lapp H, Ching KA, Block D, Zhang J, Soden R, Hayakawa M, Kreiman G, et al: A gene atlas of the mouse and human protein-encoding transcriptomes. Proc Natl Acad Sci USA 2004, 101:6062-6067.

3. Roelen BA, van Rooijen MA, Mummery CL: Expression of ALK-1, a type 1 serine/threonine kinase receptor, coincides with sites of vasculogenesis 
and angiogenesis in early mouse development. Dev Dyn 1997, 209:418-430.

4. Sanz-Rodriguez F, Fernandez LA, Zarrabeitia R, Perez-Molino A, Ramirez JR, Coto E, Bernabeu C, Botella LM: Mutation analysis in Spanish patients with hereditary hemorrhagic telangiectasia: deficient endoglin upregulation in activated monocytes. Clin Chem 2004, 50:2003-2011.

5. Konig HG, Kogel D, Rami A, Prehn JH: TGF- $\beta 1$ activates two distinct type I receptors in neurons: implications for neuronal NF- $\kappa$ B signaling. J Cell Biol 2005, 168:1077-1086.

6. Beger B, Robertson K, Evans A, Grant A, Berg J: Expression of endoglin and the activin receptor-like kinase 1 in skin suggests a role for these receptors in normal skin function and skin tumorigenesis. Brit J Dermatol 2006, 154:379-382

7. Li L, Zhao XY, Wang BE: Down-regulation of transforming growth factor beta 1/activin receptor-like kinase 1 pathway gene expression by herbal compound 861 is related to deactivation of LX-2 cells. World J Gastroenterol 2008, 14:2894-2899.

8. Finnson KW, Parker WL, ten Dijke P, Thorikay M, Philip A: ALK1 opposes ALK5/Smad3 signaling and expression of extracellular matrix components in human chondrocytes. J Bone Miner Res 2008, 23:896-906.

9. Mancini ML, Verdi JM, Conley BA, Nicola T, Spicer DB, Oxburgh LH, Vary CP: Endoglin is required for myogenic differentiation potential of neural crest stem cells. Dev Biol 2007, 308:520-533.

10. Velasco S, Alvarez-Munoz P, Pericacho M, Dijke PT, Bernabeu C, LopezNovoa JM, Rodriguez-Barbero A: L- and S-endoglin differentially modulate TGFbeta1 signaling mediated by ALK1 and ALK5 in L6E9 myoblasts. J Cell Sci 2008, 121:913-919.

11. Oh SP, Seki T, Goss KA, Imamura T, Yi Y, Donahoe PK, Li L, Miyazono K, ten Dijke $P$, Kim S, et al: Activin receptor-like kinase 1 modulates transforming growth factor-beta 1 signaling in the regulation of angiogenesis. Proc Natl Acad Sci USA 2000, 97:2626-2631.

12. Lux A, Salway F, Dressman HK, Kroner-Lux G, Hafner M, Day PJ, Marchuk DA, Garland J: ALK1 signalling analysis identifies angiogenesis related genes and reveals disparity between TGF-beta and constitutively active receptor induced gene expression. BMC Cardiovasc Disord 2006, 6:13.

13. Seki T, Yun J, Oh SP: Arterial endothelium-specific activin receptor-like kinase 1 expression suggests its role in arterialization and vascular remodeling. Circ Res 2003, 93:682-689.

14. Urness LD, Sorensen LK, Li DY: Arteriovenous malformations in mice lacking activin receptor-like kinase-1. Nat Genet 2000, 26:328-331.

15. Lux A, Attisano L, Marchuk DA: Assignment of transforming growth factor beta1 and beta3 and a third new ligand to the type I receptor ALK-1. J Biol Chem 1999, 274:9984-9992.

16. David L, Mallet C, Mazerbourg S, Feige JJ, Bailly S: Identification of BMP9 and BMP10 as functional activators of the orphan activin receptor-like kinase 1 (ALK1) in endothelial cells. Blood 2007, 109:1953-1961.

17. Bernabeu C, Lopez-Novoa JM, Quintanilla M: The emerging role of TGFbeta superfamily coreceptors in cancer. Biochim Biophys Acta 2009, 1792:954-973.

18. Lebrin F, Goumans MJ, Jonker L, Carvalho RL, Valdimarsdottir G, Thorikay M, Mummery C, Arthur HM, ten Dijke P: Endoglin promotes endothelial cell proliferation and TGF-beta/ALK1 signal transduction. EMBO J 2004, 23:4018-4028.

19. Goumans MJ, Lebrin F, Valdimarsdottir G: Controlling the angiogenic switch: a balance between two distinct TGF- $\beta$ receptor signaling pathways. Trends Cardiovas Med 2003, 13:301-307.

20. David L, Mallet C, Keramidas M, Lamande N, Gasc JM, Dupuis-Girod S, Plauchu H, Feige JJ, Bailly S: Bone morphogenetic protein-9 is a circulating vascular quiescence factor. Circ Res 2008, 102:914-922.

21. ten Dijke $P$, Ichijo H, Franzen P, Schulz P, Saras J, Toyoshima H, Heldin CH, Miyazono K: Activin receptor-like kinases: a novel subclass of cell-surface receptors with predicted serine/threonine kinase activity. Oncogene 1993, 8:2879-2887

22. Attisano L, Carcamo J, Ventura F, Weis FM, Massague J, Wrana JL: Identification of human activin and TGF beta type I receptors that form heteromeric kinase complexes with type II receptors. Cell 1993, 75:671-680.

23. McAllister KA, Grogg KM, Johnson DW, Gallione CJ, Baldwin MA, Jackson CE, Helmbold EA, Markel DS, McKinnon WC, Murrell J, et al: Endoglin, a TGF- beta binding protein of endothelial cells, is the gene for hereditary haemorrhagic telangiectasia type 1. Nat Genet 1994, 8:345-351.

24. Li DY, Sorensen LK, Brooke BS, Urness LD, Davis EC, Taylor DG, Boak BB, Wendel DP: Defective angiogenesis in mice lacking endoglin. Science 1999, 284:1534-1537.

25. Arthur HM, Ure J, Smith AJ, Renforth G, Wilson DI, Torsney E, Charlton R, Parums DV, Jowett T, Marchuk DA, et al: Endoglin, an ancillary TGFbeta receptor, is required for extraembryonic angiogenesis and plays a key role in heart development. Dev Biol 2000, 217:42-53.

26. Bourdeau A, Dumont DJ, Letarte M: A murine model of hereditary hemorrhagic telangiectasia. J Clin Invest 1999, 104:1343-1351.

27. Govani FS, Shovlin CL: Hereditary haemorrhagic telangiectasia: a clinical and scientific review. Eur J Hum Genet 2009, 17:860-871.

28. Shovlin $\mathrm{CL}$, Guttmacher AE, Buscarini E, Faughnan ME, Hyland $\mathrm{RH}$, Westermann CJ, Kjeldsen AD, Plauchu H: Diagnostic criteria for hereditary hemorrhagic telangiectasia (Rendu-Osler-Weber syndrome). Am J Med Genet 2000, 91:66-67.

29. Abdalla SA, Pece-Barbara N, Vera S, Tapia E, Paez E, Bernabeu C, Letarte M: Analysis of ALK-1 and endoglin in newborns from families with hereditary hemorrhagic telangiectasia type 2. Hum Mol Genet 2000, 9:1227-1237.

30. Rius C, Smith JD, Almendro N, Langa C, Botella LM, Marchuk DA, Vary CP, Bernabeu $\mathrm{C}$ : Cloning of the promoter region of human endoglin, the target gene for hereditary hemorrhagic telangiectasia type 1. Blood 1998, 92:4677-4690

31. Botella LM, Sanchez-Elsner T, Rius C, Corbi A, Bernabeu C: Identification of a critical Sp1 site within the endoglin promoter and its involvement in the transforming growth factor-beta stimulation. J Biol Chem 2001, 276:34486-34494.

32. Botella LM, Sanchez-Elsner T, Sanz-Rodriguez F, Kojima S, Shimada J, Guerrero-Esteo M, Cooreman MP, Ratziu V, Langa C, Vary CP, et al: Transcriptional activation of endoglin and transforming growth factorbeta signaling components by cooperative interaction between Sp1 and KLF6: their potential role in the response to vascular injury. Blood 2002, 100:4001-4010.

33. Sanchez-Elsner T, Botella LM, Velasco B, Langa C, Bernabeu C: Endoglin expression is regulated by transcriptional cooperation between the hypoxia and transforming growth factor-beta pathways. J Biol Chem 2002, 277:43799-43808.

34. Berg JN, Gallione CJ, Stenzel T, Johnson DW, Allen WP, Schwartz CE, Jackson CE, Porteous ME, Marchuk DA: The activin receptor-like kinase 1 gene: genomic structure and mutations in hereditary hemorrhagic telangiectasia type 2. Am J Hum Genet 1997, 61:60-67.

35. Goumans MJ, Valdimarsdottir $G$, Itoh $S$, Rosendahl A, Sideras $P$, ten Dijke $P$. Balancing the activation state of the endothelium via two distinct TGFbeta type I receptors. EMBO J 2002, 21:1743-1753.

36. Sanchez-Elsner T, Ramirez JR, Sanz-Rodriguez F, Varela E, Bernabeu C, Botella LM: A cross-talk between hypoxia and TGF-beta orchestrates erythropoietin gene regulation through SP1 and Smads. J Mol Biol 2004, 336:9-24.

37. Pimanda JE, Chan WY, Wilson NK, Smith AM, Kinston S, Knezevic K, Janes ME, Landry JR, Kolb-Kokocinski A, Frampton J, et al: Endoglin expression in blood and endothelium is differentially regulated by modular assembly of the Ets/Gata hemangioblast code. Blood 2008, 112:4512-4522.

38. Wasylyk B, Hagman J, Gutierrez-Hartmann A: Ets transcription factors: nuclear effectors of the Ras-MAP-kinase signaling pathway. Trends Biochem Sci 1998, 23:213-216.

39. Bloom BB, Humphries DE, Kuang PP, Fine A, Goldstein RH: Structure and expression of the promoter for the R4/ALK5 human type I transforming growth factor-beta receptor: regulation by TGF-beta. Biochim Biophys Acta 1996, 1312:243-248.

40. Lania L, Majello B, De Luca P: Transcriptional regulation by the Sp family proteins. Int J Biochem Cell B 1997, 29:1313-1323.

41. Clark SJ, Harrison J, Molloy PL: Sp1 binding is inhibited by $(m) C p(m) C p G$ methylation. Gene 1997, 195:67-71.

42. Siegfried Z, Eden S, Mendelsohn M, Feng X, Tsuberi BZ, Cedar H: DNA methylation represses transcription in vivo. Nat Genet 1999, 22:203-206.

43. Simonsson S, Gurdon J: DNA demethylation is necessary for the epigenetic reprogramming of somatic cell nuclei. Nat Cell Biol 2004, 6:984-990 
44. Cao YX, Jean JC, Williams MC: Cytosine methylation of an Sp1 site contributes to organ-specific and cell-specific regulation of expression of the lung epithelial gene t1alpha. Biochem J 2000, 350:883-890.

45. Almendro N, Bellon T, Rius C, Lastres P, Langa C, Corbi A, Bernabeu C: Cloning of the human platelet endothelial cell adhesion molecule-1 promoter and its tissue-specific expression. Structural and functional characterization. J Immunol 1996, 157:5411-5421.

46. Patterson C, Perrella MA, Hsieh CM, Yoshizumi M, Lee ME, Haber E: Cloning and functional analysis of the promoter for $\mathrm{KDR} / \mathrm{flk}-1$, a receptor for vascular endothelial growth factor. J Biol Chem 1995, 270:23111-23118.

47. Gory S, Dalmon J, Prandini MH, Kortulewski T, de Launoit Y, Huber P: Requirement of a GT box (Sp1 site) and two Ets binding sites for vascular endothelial cadherin gene transcription. J Biol Chem 1998, 273:6750-6755.

48. Zhang R, Min W, Sessa WC: Functional analysis of the human endothelial nitric oxide synthase promoter. Sp1 and GATA factors are necessary for basal transcription in endothelial cells. J Biol Chem 1995, 270:15320-15326.

49. Dube A, Akbarali Y, Sato TN, Libermann TA, Oettgen P: Role of the Ets transcription factors in the regulation of the vascular-specific Tie2 gene. Circ Res 1999, 84:1177-1185.

50. Seki T, Hong KH, Yun J, Kim SJ, Oh SP: Isolation of a regulatory region of activin receptor-like kinase 1 gene sufficient for arterial endotheliumspecific expression. Circ Res 2004, 94:e72-77.

51. Butta N, Larrucea S, Alonso S, Rodriguez RB, Arias-Salgado EG, Ayuso MS, Gonzalez-Manchon C, Parrilla R: Role of transcription factor Sp1 and CpG methylation on the regulation of the human podocalyxin gene promoter. BMC Mol Biol 2006, 7:17.

52. Korchynskyi $\mathrm{O}$, ten Dijke P: Identification and functional characterization of distinct critically important bone morphogenetic protein-specific response elements in the Id1 promoter. J Biol Chem 2002, 277:4883-4891.

53. Sanchez-Elsner T, Botella LM, Velasco B, Corbi A, Attisano L, Bernabeu C: Synergistic cooperation between hypoxia and transforming growth factor-beta pathways on human vascular endothelial growth factor gene expression. J Biol Chem 2001, 276:38527-38535.

doi:10.1186/1471-2199-11-51

Cite this article as: Garrido-Martin et al.: Characterization of the human Activin-A receptor type II-like kinase 1 (ACVRL1) promoter and its regulation by Sp1. BMC Molecular Biology 2010 11:51.

\section{Submit your next manuscript to BioMed Central and take full advantage of:}

- Convenient online submission

- Thorough peer review

- No space constraints or color figure charges

- Immediate publication on acceptance

- Inclusion in PubMed, CAS, Scopus and Google Scholar

- Research which is freely available for redistribution

Submit your manuscript at www.biomedcentral.com/submit
Biomed Central 